\title{
Mir-139-5p inhibits glioma cell proliferation and progression by targeting GABRA 1
}

\author{
Lei Wang ${ }^{1 *}$ (ㅁ, Yan Liu², Zhengtao Yu ${ }^{3}$, Jianwu Gong ${ }^{1}$, Zhiyong Deng ${ }^{1}$, Nianjun Ren ${ }^{1}$, Zhe Zhong ${ }^{1}$, Hao Cai ${ }^{1}$,
} Zhi Tang ${ }^{1}$, Haofeng Cheng ${ }^{1}$, Shuai Chen ${ }^{1}$ and Zhengwen $\mathrm{He}^{1 *}$

\begin{abstract}
Glioma is an extremely aggressive malignant neoplasm of the central nervous system. MicroRNA (miRNA) are known to bind to specific target mRNA to regulate post-transcriptional gene expression and are, therefore, currently regarded as promising biomarkers for glioma diagnosis and prognosis. The aim of the present study was to examine the pathogenesis and potential molecular markers of glioma by comparing the differential expression of miRNA and mRNA between glioma tissue and peritumor brain tissue. We explored the impact of screened core miRNA and mRNA on cell proliferation, invasion, and migration of glioma. An miRNA expression profile dataset (GSE90603) and a transcriptome profile dataset (GSE90598) were downloaded from combined miRNA-mRNA microarray chips in the Gene Expression Omnibus (GEO) database. Overall, 59 differentially expressed miRNAs (DEMs) and 419 differentially expressed genes (DEGs) were identified using the $\mathrm{R}$ limma software package. FunRich software was used to predict DEM target genes and miRNA-gene pairs, and Perl software was used to find overlapping genes between DEGs and DEM target genes. There were 129 overlapping genes regulated by nine miRNAs between target genes of the DEMs and DEGs. The Chinese Glioma Genome Atlas(CGGA) was analyzed in order to identify miRNAs with diagnostic and prognostic significance. MiR-139-5p, miR-137, and miR-338-3p were validated to be significantly linked to prognosis in glioma patients. Finally, we validated that miR-139-5p affected glioma malignant biological behavior via targeting gamma-aminobutyric acid A receptor alpha 1(GABRA1) through rescue experiments. Low miR-139-5p expression was correlated with survival probability and World Health Organization (WHO) grade. MiR-139-5p overexpression inhibited cell proliferation, migration, and invasion of glioma in vitro. GABRA1 was identified as a functional downstream target of miR-139-5p. Decreased GABRA1 expression was related to similar biological roles as miR-139-5p overexpression while upregulation of GABRA1 effectively reversed the inhibition effects of miR-139-5p. These results demonstrate a novel axis for miR-139-5p/GABRA1 in glioma progression and provide potential prognostic predictors and therapeutic target for glioma patients.
\end{abstract}

Keywords: Glioma, MiR-139-5p, GABRA1, Biomarker, Invasion, Migration, Proliferation, Apoptosis

\section{Introduction}

Glioma is the most prevalent primary malignant neoplasm in the central nervous system, with high recurrence and mortality rates. According to the World Health

\footnotetext{
*Correspondence: wangsengyi@163.com; hezhw2001@163.com ${ }^{1}$ Department of Neurosurgery, Hunan Cancer Hospital and The Affiliated Cancer Hospital of Xiangya School of Medicine, Central South University, No.283 Tongzipo road, Yuelu district, Changsha 410006, Hunan, China Full list of author information is available at the end of the article
}

Organization (WHO) grading system, glioma has been classified into four grades [1]. The higher the grade of the glioma, the worse the prognosis of the patient. Glioblastoma multiforme(GBM), represents the most aggressive glioma subtype, with a five-year survival rate of less than $3 \%$ and average survival of less than 12 months [2]. Treatment involves surgical resection followed by adjuvant chemotherapy or concurrent chemoradiotherapy [3]. However, the 3-year survival rate remains at a low level, 
especially for GBM patients, with the 3-year survival rate only slightly increasing from $2.00 \%-5.00 \%$ to $7.31 \%$ [4]. Therefore, researchers have turned their attention to study the potential molecular mechanisms of GBM, and to find novel prognostic biomarkers for the early diagnosis and monitoring of tumorigenesis and for evaluating prognosis.

MicroRNAs (miRNA) are commonly defined as noncoding RNAs consisting of 18-25 nucleotides that regulate post-transcriptional gene expression via specific binding to target mRNA. MiRNA are known to be involved in the regulation of tumor cellular physiological processes, and the aberrant expression and crossregulation of miRNA and target mRNA can be applied as a potential diagnostic and prognostic biomarker in all grades of glioma. Researchers have begun identifying miRNAs that may act as glioma oncogenes or suppressor genes, which could then be applied as diagnostic and predictive biomarkers, and even act as molecular therapeutic targets against glioma $[5,6]$.

To identify the novel diagnostic and predictive value of miRNAs as biomarkers, bioinformatics tools were used to integrate analysis of differential expression of
miRNAs and mRNAs from paired 16 fresh-frozen GBM samples and seven peritumoral tissues. Via integrating the miRNA and mRNA expression in the GBM pairs, we identified a number of predictive miRNAs that can be used as biomarkers for diagnosis and prognosis of GBM, and which could then be used as potential clinical therapeutic targets (flow chart see Fig. 1). Specifically, we identified that miR-139-5p and GABRA1 served as inverse agents in the regulation of malignant phenotypes of glioma cells, we doubted whether the function of miR-139-5p on glioma cells was mediated through its inhibitory impression on GABRA1 expression. We, performed rescue experiments to dispose it. In this study, we firstly provide a series of potential targets for future investigation into the molecular mechanisms involved in glioma and offer insight into miRNAs as biomarkers for disease progression. Furthermore, we explore the possible role of miR-139-5p and GABRA1 in the development of glioma and the underlying molecular regulation mechanism. Our findings may provide new insights for the diagnosis and treatment of glioma.

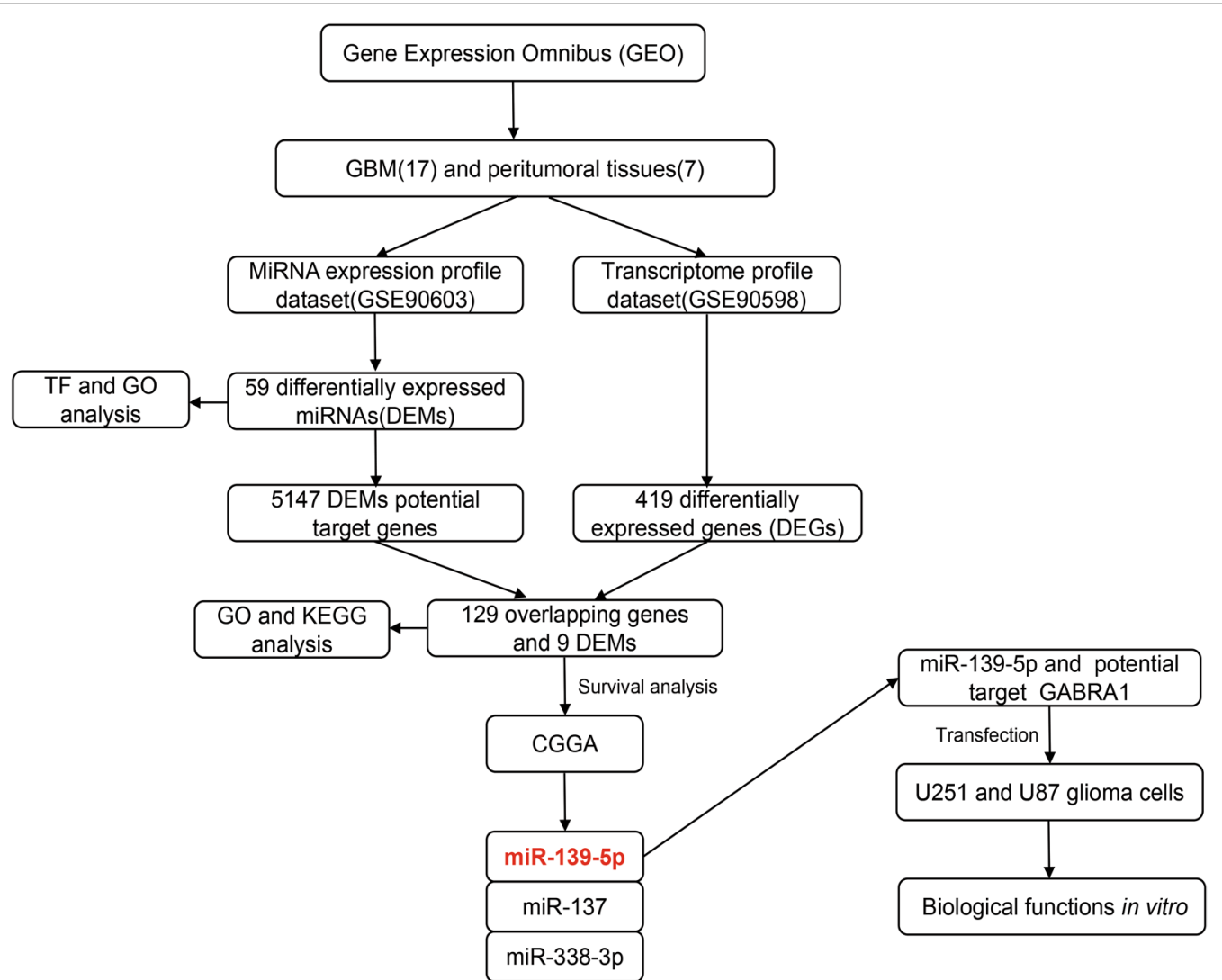

Fig. 1 This figure presents an overview of the analysis workfow 


\section{Methods}

\section{Microarray data}

GSE90603 and GSE90598 were downloaded from the GEO database. A total of 23 fresh-frozen samples were analyzed, including 16 individual samples of GBM and seven individual samples of healthy brain tissues. The expression of miRNA (GSE90603) was detected using the platform GPL21582 (Affymetrix Multispecies miRNA-4 Array). The mRNA dataset(GSE90598) was analyzed by using the platform GPL17692 (Affymetrix Human Gene 2.1 ST Array).

\section{Identification of DEMs and DEGs}

The DEMs and DEGs between GBM samples and healthy brain tissues was processed using $\mathrm{R}$ software. The Affy $R$ package was used to process the downloaded raw data and the limma $\mathrm{R}$ package was then used to identify DEMs and DEGs. The adjusted $p$ values were used to decrease the false positive rate using Benjamini and Hochberg false discovery rate method by default. $|\log 2 \mathrm{FC}| \geq 2$ and adjust $p<0.05$ were identified as the DEMs or DEGs between GBM sample and healthy brain tissues.

\section{Gene, function and pathway enrichment analysis}

FunRich is a stand-alone software tool used mainly for functional enrichment and interaction network analysis of genes and proteins (http://www.funrich.org) [7]. FunRich was used to perform DEM downstream transcription factor analysis and DEM target genes prediction. In addition, GO function and KEGG pathway were analyzed using the FunRich software. The core miRNAs and their target genes regulatory network was constructed and visualized by Cytoscape 3.6.1 software.

\section{Survival analysis and glioma grading analysis}

The Chinese Glioma Genome Altas (CGGA) database is a user-friendly web application for data storage and analysis to explore brain tumors datasets over 2,000 samples from Chinese cohorts. This database includes miRNA microarray (198 samples) and matched clinical data and could used for assessing the effect of certain miRNA on glioma patients survival. In addition, the miRNA expression level between grade II and grade IV glioma was analyzed via CGGA database. The median expression values of every DEMs in glioma samples were calculated, based on which they were divided into high (above median)-and low-expression(below median) groups. Integrated with the prognostic outcomes, including the overall survival, obtained from CGGA database, the Kaplan-Meier survival curves of these two groups were plotted and the log-rank tests were used to assess the relationship of gene expression to survival. A $p$-value $<0.05$ was set as the threshold for statistical significance.

\section{Cell culture}

Human brain glioma cell lines U251 and U87 were procured from BeNa Culture Collection (BNCC, China). Cell lines were cultivated in Dulbecco's Modified Eagle's Medium (DMEM, Sigma, USA) with $10 \%$ foetal bovine serum (FBS, Gibco, USA) in $5 \% \mathrm{CO}_{2}$ at $37^{\circ} \mathrm{C}$.

\section{Cell transfection}

Lipofectamine 2000 (LIP2000, invitrogen, USA) was applied to transiently transfect the miR-139-5p mimics or inhibitor as well as the GABRA1 overexpression vector or GABRA1 siRNA into glioma cells according to the manufacture's instruction. MiR-139-5p mimic, miRNA mimic negative control (mimic NC), miR-139-5p inhibitor control, miRNA inhibitor negative control (inhibitor NC) were chemically synthesized by HonorGene (China). GABRA1 overexpression plasmid and siRNA targeting GABRA1 were purchased from RiboBio (China).

\section{Quantitative Real time-PCR (qRT-PCR)}

TRIzol Reagent (Thermo, USA) was applied to extract the total RNA from samples according to the manufacture's recommendation. The reverse transcription of $200 \mathrm{ng}$ total RNA was utilizing SuperRT RT reagent Kit (CWBio, China) and quantitative real-time PCR with SYBR PCR Master Mix(CWBio, China). The PCR was set at the initial denaturation of $10 \mathrm{~min}$ at $95^{\circ} \mathrm{C}$, following with $15 \mathrm{~s}$ at $95^{\circ} \mathrm{C}$, and $30 \mathrm{~s}$ at $60{ }^{\circ} \mathrm{C}$ in a total of 40 cycles. All experiments were carried out in triplicate. The miR-139-5p expression was normalized to U6 while the GABRA1 was normalized to actin. The relative expression ratios of genes were calculated by the $2-\Delta \Delta \mathrm{CT}$ method. The primers involved in this assay were shown as follow:

hsa-miR-139-5p: TCTACAGTGCACGTGTCTCC; U6, F: CTCGCTTCGGCAGCACA, R:AACGCTTCA CGAATTTGCGT; actin, F:ACCCTGAAGTACCCCATC GAG, R:AGCACAGCCTGGATAGCAAC; GABRA1, F:ATGATGGAGCTCGAGGCAAA, R:AGCTCTGAA TTGTGCTGGGT. 


\section{Western blot assay}

Total protein in cells was collected, and the concentration of protein wasquantified with Pierce BCA Protein Assay Kit. After segregated by $10 \%$ sodium dodecyl sulphate-polyacrylamide gel electrophoresis (SDS-PAGE) gel, extracted proteins were transferred to polyvinylidene difluoride (PVDF) membranes and blocked in Tris-buffered saline-Tween with $5 \%$ skim milk at room temperature for $60 \mathrm{~min}$. Western blot analysis was performed according to standard procedures. Primary antibodies were GABRA1 (1:1000, proteintech, USA), $\beta$-actin (1:5000, proteintech, USA), and corresponding secondary antibodies were anti-mouse and anti-rabbit(proteintech, USA). X-ray film was pressed in dark. Finally, the proteins were detected by enhanced chemiluminescence (ECL), followed by expose about $60 \mathrm{~s}$ for scanning and measuring.

\section{EdU assay}

The transfected glioma cells were seeded onto the sixwell tissue-culturing plates $\left(1 \times 10^{5}\right.$ cells per well). Following the indicated treatments an EdU Apollo-567 assay kit (RiboBio, China) was utilized to test cell proliferation, with nuclear EdU and DAPI staining visualized under a fluorescent microscope.

\section{Cell apoptosis assay by flow cytometry}

Apoptotic cells were tested by apoptosis detection kit (KeyGen BioTECH, China) according to the manufacturer's instructions. Cells under log phase were obtained. $500 \mu \mathrm{l}$ binding buffer was used to resuspend cells after cells were collected. Cells were added with isometric 5 $\mu \mathrm{l}$ Annexin V-APC and $5 \mu \mathrm{l}$ propidium iodide and incubated for $10 \mathrm{~min}$ at room temperature in the dark. Cells were tested using the flow cytometry.

\section{Wound-healing assay}

At $6 \mathrm{~h}$ post-transfection, the cells were digested, centrifuged and re-suspended in FBS-free culture. The concentration of cells was adjusted to $5 \times 10^{5}$ cells $/ \mathrm{ml}$. Then cell layers were scratched with a $100 \mu$ l sterile pipette tip. After removing cell culture medium and suspension cells and cell debris, each well was added with serumfree medium and stored in incubator for 24 and $48 \mathrm{~h}$. Cell migration area was then viewed and photographed after incubation for $24 \mathrm{~h}$. Meanwhile, scratch test was performed to evaluate the difference in cell healing ability according to the migration area.

\section{Transwell assay}

The Matrigel (BD, USA) melted at $4{ }^{\circ} \mathrm{C}$ overnight. $100 \mu \mathrm{l}$ of diluted matrigel was then added in the chamber. Afterwards, $200 \mu \mathrm{l}$ of serum-free medium was added to the upper chamber; meanwhile, $500 \mu \mathrm{l}$ of $10 \%$ FBS DMEM was added to the lower chambers, and $2 \times 10^{5}$ collected cells in total were planted in the upper ones and cultivated in the incubator for another $48 \mathrm{~h}$. Subsequently, the invading chamber was taken out, and cells on the polycarbonate membrane were fixed with $4 \%$ paraformaldehydel, followed by staining with $0.1 \%$ crystal violet. Three random fields were selected, and invaded cells were counted under a microscope. The experiments were carried out in triplicate.

\section{Statistical analysis}

All data are presented as mean \pm SD of three independent experiments. Comparisons between the quantitative data were made using Student's t test, with $p<0.05$ considered statistically significant. Survival rate was delineated using Kaplan-Meier method. Statistical and graphical analyses were performed with the use of GraphPad Prism 5 software.

Table 1 Top 20 DEMs between GBM sample compared with normal brain tissues

\begin{tabular}{lcl}
\hline ID & logFC & adj.P.Val \\
\hline hsa-miR-320d & 2.226057281 & $4.84 \mathrm{E}-07$ \\
hsa-miR-455-3p & 2.521827549 & $4.84 \mathrm{E}-07$ \\
hsa-miR-25-3p & 3.144720167 & $5.08 \mathrm{E}-07$ \\
hsa-miR-500a-5p & 2.346944027 & $9.88 \mathrm{E}-07$ \\
hsa-miR-28-3p & 2.748360285 & $1.68 \mathrm{E}-06$ \\
hsa-miR-106b-3p & 2.745815992 & $2.73 \mathrm{E}-06$ \\
hsa-miR-362-5p & 2.202213072 & $2.79 \mathrm{E}-06$ \\
hsa-miR-155-5p & 3.886573305 & $4.04 \mathrm{E}-06$ \\
hsa-miR-490-5p & -2.531780737 & $5.53 \mathrm{E}-06$ \\
hsa-miR-21-5p & 4.138010995 & $7.73 \mathrm{E}-06$ \\
hsa-miR-338-3p & -2.728425739 & $7.73 \mathrm{E}-06$ \\
hsa-miR-424-3p & 2.83234903 & $1.17 \mathrm{E}-05$ \\
hsa-miR-23a-3p & 2.602961549 & $1.24 \mathrm{E}-05$ \\
hsa-miR-18a-5p & 2.111435648 & $1.52 \mathrm{E}-05$ \\
hsa-miR-339-5p & 2.623198868 & $1.97 \mathrm{E}-05$ \\
hsa-miR-15b-5p & 3.178531266 & $1.97 \mathrm{E}-05$ \\
hsa-miR-6872-3p & 2.196366981 & $2.41 \mathrm{E}-05$ \\
hsa-miR-93-5p & 2.182576627 & $2.75 \mathrm{E}-05$ \\
hsa-mir-92b & 2.64699354 & $3.27 \mathrm{E}-05$ \\
hsa-miR-181a-2-3p & 2.532050498 & $3.60 \mathrm{E}-05$ \\
\hline
\end{tabular}

$D E M s$ differentially expressed miRNAs, GBM glioblastoma 
Table 2 Top 20 DEGs between GBM sample compared with normal brain tissues

\begin{tabular}{lll}
\hline ID & logFC & adj.P.Val \\
\hline SEC14L5 & -2.410823841 & $2.49 \mathrm{E}-09$ \\
KCNT1 & -2.989158475 & $2.49 \mathrm{E}-09$ \\
PEX5L & -3.806308716 & $2.33 \mathrm{E}-08$ \\
LGI3 & -3.224367902 & $3.81 \mathrm{E}-08$ \\
OPALIN & -4.148538323 & $3.81 \mathrm{E}-08$ \\
CORO6 & -2.317401319 & $3.81 \mathrm{E}-08$ \\
GABRG1 & -4.377954642 & $4.80 \mathrm{E}-08$ \\
KCNH3 & -2.635371133 & $6.24 \mathrm{E}-08$ \\
ROGDI & -2.067007874 & $1.02 \mathrm{E}-07$ \\
GRM3 & -3.433516194 & $1.08 \mathrm{E}-07$ \\
UNC13C & -4.564034026 & $1.08 \mathrm{E}-07$ \\
RASGRF1 & -2.865726351 & $1.12 \mathrm{E}-07$ \\
CABP1 & -3.404983957 & $1.25 \mathrm{E}-07$ \\
PXDN & 2.869541549 & $1.79 \mathrm{E}-07$ \\
SH3GL3 & -3.060067098 & $2.41 \mathrm{E}-07$ \\
PPP2R2C & -2.912293455 & $2.57 \mathrm{E}-07$ \\
RAPGEF5 & -2.685003832 & $2.85 \mathrm{E}-07$ \\
RPH3A & -3.228432626 & $2.87 \mathrm{E}-07$ \\
NECAB1 & -4.029953556 & $4.08 \mathrm{E}-07$ \\
ANK3 & -2.985792673 & $4.73 \mathrm{E}-07$ \\
\hline DEGs & &
\end{tabular}

DEGs differentially expressed genes, GBM glioblastoma

\section{Results}

Identification of differentially expressed miRNAs (DEMs) and differentially expressed genes (DEGs) in GBM Using the GSE90603 dataset, 59 DEMs were identified in the GBM samples compared with the peritumoral tissues, of which 37 were upregulated and 22 were downregulated (Additional file 1). The 20 DEMs with the lowest $p$-value are presented in Table 1 . In total, 419 DEGs were obtained in the GSE90598 dataset, and among these, 77 were upregulated and 342 were downregulated (Additional file 2). The top 20 DEGs with the lowest $p$-value are presented in Table 2. Heatmaps of DEMs and DEGs are shown in Fig. 2.

\section{Transcription factor (TF) and gene ontology (GO) analyses of DEMs}

TF and GO analyses of DEMs were performed by FunRich software. The top ten most commonly expressed
TFs associated with DEMS were RORA, RREB1, ZFP161, FOXA1, MEF2A, NKX6-1, POU2F1, SP4, SP1, and EGR1 (Fig. 3a). GO enrichment analysis of the top ten DEMs was also performed using FunRich software. The result revealed that the majority of DEMs in the biological process (BP) category were enriched in "signal transduction", "cell communication", "nucleoside, nucleotide and nucleic acid metabolism", and "transport" (Fig. 3b and Additional file 3). The GO cellular component $(\mathrm{CC})$ enrichment analysis revealed that the majority of DEMs in this category were enriched in "nucleus", "cytoplasm", "lysosome", and "golgi apparatus" (Fig. 3c and Additional file 4). In the molecular function (MF) category, the majority of DEMs were associated with "transcription factor activity", "transcription regulator activity", "protein serine/threonine kinase activity", "ubiquitin-specific protease activity", "receptor signaling, and complex scaffold activity" (Fig. 3d and Additional file 5).

\section{DEMs target gene prediction}

The potential target genes (PTGs) of each DEM were obtained by using FunRich software. All 59 DEMs were selected for PTG prediction and 34 DEMs were identified. The FunRich tool generated 5147 PTGs for 34 DEMs. We then took the overlapping gene intersection of PTGs and DEGs, and analyzed the correlation of downregulated miRNAs with upregulated genes, upregulated miRNAs, and downregulated genes. There were nine core DEMs and 129 overlapping genes with a targeted relationship (Table 3 ).

\section{MiRNA-gene regulatory network construction}

According to the information obtained from the nine core DEMs and 129 overlapping target genes, core DEMs and target gene regulatory networks were constructed and visualized using Cytoscape software (Fig. 4). Overall, the data indicate that the nine core DEMs and 129 overlapping target genes may play important roles in the diagnosis and prognosis of glioma.

GO and Kyoto Encyclopedia of Genes and Genomes(KEGG) pathway enrichment analysis of overlapping target genes

To explore roles that overlapping target genes paly in biological functions, GO and KEGG pathway analysis were performed. The overlapping genes were significantly enriched in BP, including "synaptic vesicle cycle", 


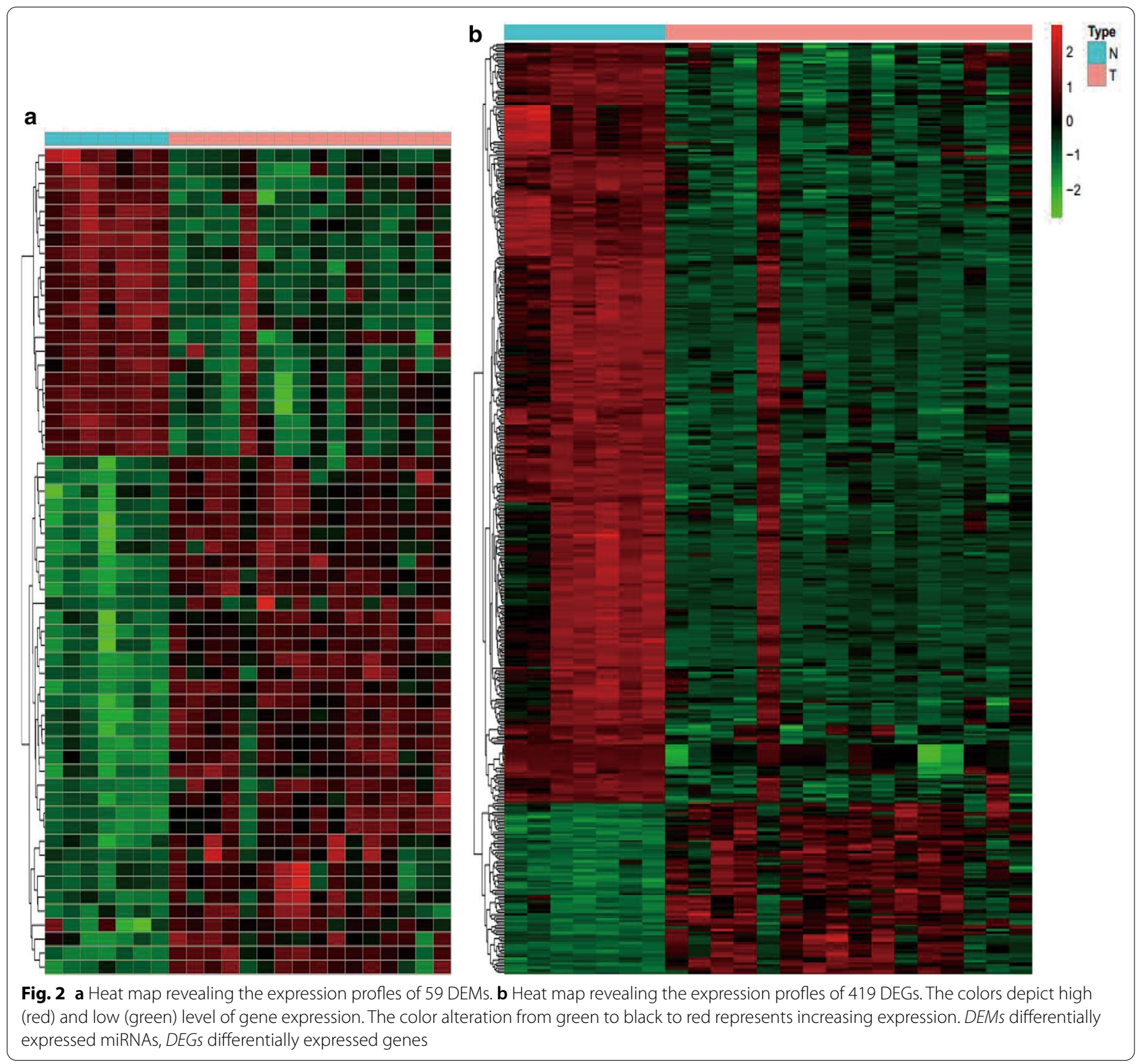

"regulation of membrane potential", "glutamate receptor signaling pathway", "modulation of chemical synaptic transmission" and so on (Fig. 5a and Additional file 6). For MF, The overlapping target genes were mainly related to "ion gate channel activity", "gated channel activity", "ion channel activity", "substrate-specific channel activity" and so on (Fig. 5a and Additional file 6). In addition, $\mathrm{CC}$ analysis indicated that the overlapping target genes were involved in "presynapse", "glutamatergic synapse", "synaptic membrane", "presynaptic membrane" and so on (Fig. 5a Additional file 6). KEGG analysis revealed that the overlapping target genes were mostly enriched in "glutamatergic synapse", "dopaminergic synapse", "retrograde endocannabinoid signaling", "nicotine addiction" and "synaptic vesicle cycle" (Fig. 5b and Additional file 7). 


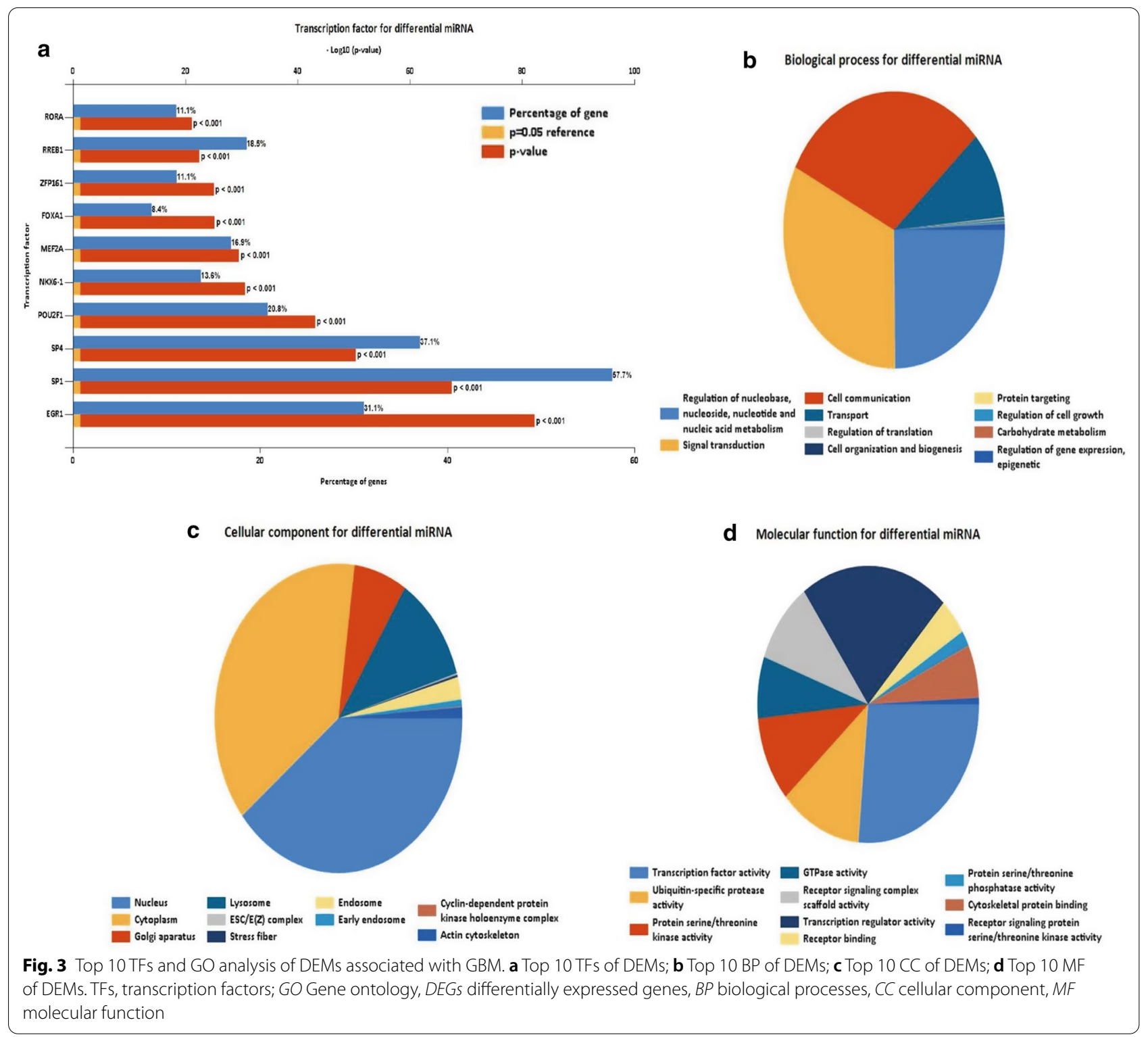

\section{Survival analysis}

The relationship between the expression of the nine core DEMs (miR-338-3p, miR-137, miR-128-3p, miR218-5p, miR-7-5p, miR-139-5p, miR-124-3p, miR383-5p, and miR-138-5p) and the overall survival of patients with all grades of glioma was analyzed using the Chinese Glioma Genome Atlas (CGGA). Only three miRNA clinical datasets existed in the CGGA database (miR-137, miR-139-5p, and miR-338-3p). We found that the high expression of miR-137 and miR338-3p were associated with poor overall survival rate in patients with all grades of glioma (Fig. 6a, b; $p<0.01$ ) while low expression of miR-139-5p was associated with poor overall survival rates (Fig. $6 c ; p<0.01$ ).

To reveal the correlation of core miRNA expression profiles with glioma grade, we compared core miRNAs distribution data of grade II-IV cases obtained in the CGGA database. The expression of miR-137 and 
Table 3 Overlapping genes between DEGs and DEMs target genes

\begin{tabular}{|c|c|c|c|c|}
\hline miRNA & mRNA & Target & mirnaLogFC & mrnaLogFC \\
\hline hsa-miR-338-3p & MYT1L & Target & -2.728425739 & 3.946 \\
\hline hsa-miR-338-3p & RGS7BP & arget & -2.728425739 & 4.314153573 \\
\hline hsa-miR-137 & ANO4 & Target & -2.997290651 & 2.895129398 \\
\hline hsa-miR-137 & ATP1B1 & Target & -2.997290651 & 6.271266512 \\
\hline hsa-miR-137 & C11orf87 & Target & -2.997290651 & 3.557787858 \\
\hline nsa-miR-137 & CEND1 & & -2.997 & 6671 \\
\hline hsa-miR-137 & $\mathrm{CHGA}$ & Target & -2.997290651 & 43461 \\
\hline hsa-miR-137 & DIRAS2 & Target & -2.997290651 & 4.112396985 \\
\hline hsa-miR-137 & EDIL3 & Target & -2.997290651 & 5.764240871 \\
\hline hsa-miR-137 & $\mathrm{EZH} 2$ & Target & -2.997290651 & 4.634117944 \\
\hline nsa-miR-137 & GABRA1 & & -2.997290651 & 4605 \\
\hline hsa-m & & & & \\
\hline hsa-miR-137 & HLF & Target & -2.9972 & 9022 \\
\hline hsa-miR-137 & NETO1 & Target & -2.997290651 & 4.134785091 \\
\hline hsa-miR-137 & NRXN1 & Target & -2.997290651 & 5.784845966 \\
\hline hsa-miR-137 & NRXN3 & Target & -2.997290651 & 3.652567346 \\
\hline רsa-miR-137 & PLCB1 & & -2 & 5061 \\
\hline hsa-miR-137 & PTGFRN & get & -2 & 013 \\
\hline hsa-miR-137 & PTPN5 & Target & -2.997290651 & 043 \\
\hline hsa-miR-137 & RAPGEF5 & Target & -2.997290651 & 4.030838556 \\
\hline hsa-miR-137 & RCAN2 & Target & -2.9 & 37004 \\
\hline hsa-miR-137 & RGS7BP & rget & -2.9 & 3573 \\
\hline hsa-miR-137 & ST18 & Target & -2.997290651 & 3.050911173 \\
\hline hsa-miR-137 & SYT1 & Target & -2.997290651 & 4.165893064 \\
\hline hsa-miR-137 & TNC & Target & -2.99729 & 6272 \\
\hline hsa-miR-137 & UNC79 & et & -2 & 0236 \\
\hline hsa-miR-137 & WIF1 & yet & -2 & 045 \\
\hline hsa-miR-128-3p & CABP1 & & -2.521510423 & 3.190706419 \\
\hline hsa-miR-128-3p & DIRAS2 & Target & -2.521510423 & 4.112396985 \\
\hline hsa-miR-128-3p & ERC2 & Target & -2.521510423 & 4.094505701 \\
\hline hsa-miR-128-3p & JAG1 & Target & -2.521510423 & 6.666037367 \\
\hline hsa-miR-128-3p & KLHDC8A & Target & -2.521510423 & 5.045962448 \\
\hline hsa-miR-128-3p & PPFIA2 & Target & & 4.623994699 \\
\hline hsa-miR-128-3p & PTPN5 & Target & -2.521510423 & 3.471111043 \\
\hline hsa-miR-128-3p & SAMD9L & Target & -2.521510423 & 5.284575791 \\
\hline hsa-miR-128-3p & SNAP25 & Target & -2.521510423 & 6.690762972 \\
\hline hsa-miR-128-3p & UGT8 & Target & -2.521510423 & 5.440745657 \\
\hline hsa-miR-128-3p & UNC13C & Target & -2.521510423 & 3.409978716 \\
\hline hsa-miR-218-5p & CELF4 & Target & -2.721703277 & 3.962404699 \\
\hline hsa-miR-218-5p & DIRAS2 & Target & -2.721703277 & 4.112396985 \\
\hline hsa-miR-218-5p & DLG2 & Target & -2.721703277 & 4.123750776 \\
\hline hsa-miR-218-5p & ERC2 & Target & -2.721703277 & 4.094505701 \\
\hline hsa-miR-218-5p & GABRB2 & Target & -2.721703277 & 3.053748336 \\
\hline hsa-miR-218-5p & GNAO1 & Target & -2.721703277 & 7.321852005 \\
\hline hsa-miR-218-5p & GNG3 & Target & -2.721703277 & 5.769726039 \\
\hline hsa-miR-218-5p & GRM3 & Target & -2.721703277 & 4.486725834 \\
\hline hsa-miR-218-5p & HLF & Target & -2.721703277 & 5.341249022 \\
\hline hsa-miR-218-5p & HS6ST3 & Target & -2.721703277 & 2.808269582 \\
\hline hsa-miR-218-5p & KCNT1 & Target & -2.721703277 & 3.426516123 \\
\hline
\end{tabular}

Table 3 (continued)

\begin{tabular}{|c|c|c|c|c|}
\hline miRNA & mRNA & Target & mirnaLogFC & mrnaLogFC \\
\hline sa-miR-218-5p & KLHDC8A & et & -2 & 5 \\
\hline$a-m i R-218-5 p$ & MYT1L & et & -2.7 & 602 \\
\hline $218-5 p$ & NECAB1 & et & -2 & 563 \\
\hline hsa-miR-218-5p & NRXN1 & Target & -2.7 & 5966 \\
\hline hsa-miR-218-5p & NRXN3 & Target & -2.721703277 & 3.652567346 \\
\hline hsa-miR-218-5p & PEX5L & get & -2.721703277 & 3.759139098 \\
\hline sa-miR-218-5p & PPP2R2C & & -2.72 & \\
\hline sa-miR-218-5p & RAP1GAP & Target & -2.7 & 598 \\
\hline sa-miR-218-5p & RAPGEF4 & Target & -2.721703277 & 5022 \\
\hline sa-miR-218-5p & SCN2B & Target & -2.721703277 & 4656 \\
\hline sa-miR-218-5p & SERPINI1 & & -2.7 & \\
\hline sa-miR-218-5p & STXBP1 & & & \\
\hline sa-miR-218-5p & TNC & & -2 & 272 \\
\hline hsa-miR-218-5p & UGT8 & Target & -2.721703277 & 5.440745657 \\
\hline hsa-miR-7-5p & ATP2B2 & Target & -3.075240145 & 4.586872611 \\
\hline sa-miR-7-5p & GABRA1 & get & -3 & \\
\hline & HCN1 & & & \\
\hline sa-miR-7-5p & HPCAL4 & & & \\
\hline$-7-5 p$ & NECAB1 & & & 4.155553563 \\
\hline hsa-miR-7-5p & RAB11FIP4 & Target & -3.075240145 & 4.429233935 \\
\hline רsa-miR-7-5p & RBFOX3 & Target & -3.075240145 & 57069 \\
\hline hsa-miR-7-5p & RGS7BP & yet & -3 & 573 \\
\hline$-7-5 p$ & S & & & \\
\hline$a-m i R-139-5 p$ & GABR/ & t & & 605 \\
\hline sa-miR-124-3p & ANO5 & et & -3.8 & 1615 \\
\hline hsa-miR-124-3p & ASPA & get & -3 & 3.84 \\
\hline hsa-miR-124-3p & BSN & Target & 939565 & 4.266708475 \\
\hline hsa-miR-124-3p & C11orf87 & Target & -3.819939565 & 3.557787858 \\
\hline hsa-miR-124-3p & C1QL3 & get & & 3.264695246 \\
\hline sa-miR-124-3p & CADM2 & & -3 & 413 \\
\hline nsa-miR-124-3p & CBLN2 & et & -3 & 923 \\
\hline hsa-miR-124-3p & CCL2 & Target & -3 & 632 \\
\hline hsa-miR-124-3p & $\mathrm{CDH} 9$ & Target & -3.819939565 & 2.597421507 \\
\hline hsa-miR-124-3p & CNTN1 & Target & -3.819939565 & 5.503812542 \\
\hline hsa-miR-124-3p & COL4A1 & Target & -3.819939565 & 4.774842046 \\
\hline hsa-miR-124-3p & DLGAP2 & Target & -3.819939565 & 2.690594307 \\
\hline hsa-miR-124-3p & $\mathrm{EZH} 2$ & Target & -3.819939565 & 4.634117944 \\
\hline hsa-miR-124-3p & FAM171A1 & Target & -3.819939565 & 5.410041936 \\
\hline hsa-miR-124-3p & FRRS1L & & -3.819939565 & 4.044676543 \\
\hline hsa-miR-124-3p & JAG1 & Target & -3.819939565 & 6.666037367 \\
\hline hsa-miR-124-3p & JAKMIP1 & Target & -3.819939565 & 3.090572083 \\
\hline hsa-miR-124-3p & KCNA1 & Target & -3.819939565 & 3.007882911 \\
\hline hsa-miR-124-3p & KCNJ3 & Target & -3.819939565 & 3.130437439 \\
\hline hsa-miR-124-3p & KIF5A & Target & -3.819939565 & 6.64331389 \\
\hline hsa-miR-124-3p & LAMC1 & Target & -3.819939565 & 5.46622199 \\
\hline hsa-miR-124-3p & LRRC7 & Target & -3.819939565 & 3.916089849 \\
\hline hsa-miR-124-3p & MAP7 & Target & -3.819939565 & 5.190958174 \\
\hline hsa-miR-124-3p & NAMPT & Target & -3.819939565 & 4.854083944 \\
\hline hsa-miR-124-3p & NEFM & Target & -3.819939565 & 3.574096381 \\
\hline
\end{tabular}


Table 3 (continued)

\begin{tabular}{|c|c|c|c|c|}
\hline miRNA & mRNA & Target & mirnaLogFC & mrnaLogFC \\
\hline hsa-miR-124-3p & NEGR1 & rget & -3.8 & 38 \\
\hline hsa-miR-124-3p & NID1 & Target & -3.819939565 & 5.602483311 \\
\hline a-miR-124-3p & NKAIN2 & rget & -3.8 & 449 \\
\hline hsa-miR-124-3p & PALM & Target & -3.81 & 5.252 \\
\hline hsa-miR-124-3p & PLCB1 & Target & -3.819 & 5.750675061 \\
\hline hsa-miR-124-3p & PTGFRN & Target & -3.819939565 & 5.222125013 \\
\hline hsa-miR-124-3p & SLC7A14 & Target & -3.819939565 & 3.168925912 \\
\hline sa-miR-124-3p & STEAP3 & Target & -3.819939565 & 733 \\
\hline sa-miR-124-3p & TBR1 & & & \\
\hline hsa-miR-124-3p & UGT8 & rget & -3.81 & 5.440745657 \\
\hline hsa-miR-124-3p & VCAN & Target & -3.819939565 & 6.729783912 \\
\hline רsa-miR-383-5p & MAL2 & Target & -3.452155995 & 2.662950301 \\
\hline רsa-miR-383-5p & PPFIA2 & Target & -3.452155995 & 4.623994699 \\
\hline isa-miR-383-5p & VEGFA & Target & -3.452155995 & 6.507050912 \\
\hline hsa-miR-138-5p & DGKE & get & -2.820786706 & 2.844643402 \\
\hline hsa-miR-138-5p & $\mathrm{EZH} 2$ & Target & -2.820786706 & 4.634117944 \\
\hline רsa-miR-138-5p & HS6ST3 & arget & -2.8207 & 2.808269582 \\
\hline רa-miR-138-5p & MGAT5B & Target & 786706 & 3.750177501 \\
\hline hsa-miR-138-5p & NETO1 & Target & 0786706 & 4.134785091 \\
\hline hsa-miR-138-5p & NPTX1 & Target & -2.820786706 & 3.918195841 \\
\hline hsa-miR-138-5p & PLLP & Target & -2.820786706 & 4.91045837 \\
\hline hsa-miR-138-5p & PTGFRN & Target & -2.820786706 & 5.222125013 \\
\hline hsa-miR-138-5p & RCAN2 & Target & -2.820786706 & 5.450737004 \\
\hline hsa-miR-138-5p & RIMS2 & Target & -2.820786706 & 3.387452792 \\
\hline hsa-miR-138-5p & SCN3B & Target & -2.820786706 & 4.019176399 \\
\hline hsa-miR-138-5p & SH3GL2 & Target & -2.820786706 & 3.166496716 \\
\hline hsa-miR-138-5p & SLC17A7 & Target & -2.820786706 & 4.686584977 \\
\hline hsa-miR-138-5p & SLC6A17 & Target & -2.820786706 & 4.443401567 \\
\hline hsa-miR-138-5p & SNAP25 & Target & -2.820786706 & 6.690762972 \\
\hline hsa-miR-138-5p & SRRM4 & Target & -2.820786706 & 3.659380613 \\
\hline hsa-miR-138-5p & WEE1 & Target & -2.820786706 & 4.41487692 \\
\hline
\end{tabular}

DEMs differentially expressed miRNAs, DEGs differentially expressed genes

miR-338-3p was positively correlated with glioma grade (Fig. 6d, e), whereas the expression of miR-139-5p was negatively correlated with glioma grade (Fig. 6f).

\section{GABRA1 as the functional target of miR-139-5p}

Combining the literature search and the bioinformatics analyses, miR-139-5p and its potential target, GABRA1, were selected for further analysis. We next aimed to ascertain the underlying molecular mechanisms by which miR-139-5p exerted its tumor suppressing roles in glioma. Hence, an miR-139-5p mimic and inhibitor were respectively transfected into the U251 and U87 cell lines. The efficacy of transfection was determined by RT-PCR, with results indicating that the relative expression of miR-139-5p was significantly increased in U251 and U87 cells after transfection of the miR-139-5p mimic (Fig. 7a). Conversely, miR-139-5p expression was dramatically downregulated after transfection with the miR-139-5p inhibitor (Fig. 7a).

Both the previous bioinformatics analyses and online miRNA database analysis (Target Scan) indicated that GABRA1 could be a target of miR-139-5p, according to the putative target sequence in the GABRA1 3'Untranslated Regions (UTR) (Fig. 7b). RT-PCR and western blot analyses were carried out to further identify whether GABRA1 expression was indeed regulated by miR139-5p. The results indicated that the mRNA and protein levels of GABRA1 were significantly increased after transfection with the miR-139-5p inhibitor, while the levels were reduced after transfection with the miR-139-5p mimics, in U251 and U87 cells (Fig. 8a-e). In addition, the results of RT-PCR assays indicated that the overexpression of GABRA1 had no effect on the expression of miR-139-5p (Fig. 8f). These results suggested that miR139-5p could directly target GABRA1 and negatively regulate its expression.

\section{MiR-139-5p regulates glioma cell migration and invasion through targeting of GABRA1.}

We next performed transwell assays and wound healing assays to evaluate the effects of miR-139-3p on the migratory and invasive capabilities of U251 and U87 cells. Both transwell and wound healing assays indicated that miR-139-5p significantly repressed U251 and U87 cell migration and invasion (Figs. 9 and 10). Lossof-function experiments suggested that the miR-139-5p inhibitor elevated U251 and U87 cell migration and invasion (Figs. 9 and 10).

Next, we explored whether GABRA1 mediated the effects of miR-139-5p on the cell migration and invasion of U251 and U87 cells. Specifically, rescue experiments were carried out by co-transfecting the miR-139-5p mimic/inhibitor with or without GABRA1 overexpression (OE) and silencing ( $\mathrm{Si}$ ). The miR-139-5p inhibitor resulted in a significant increase in the migration and invasion, while miR-139-5p mimic resulted in decreased migration and invasion, of glioma cells. OEGABRA1 remarkably rescued the suppressive effects of 


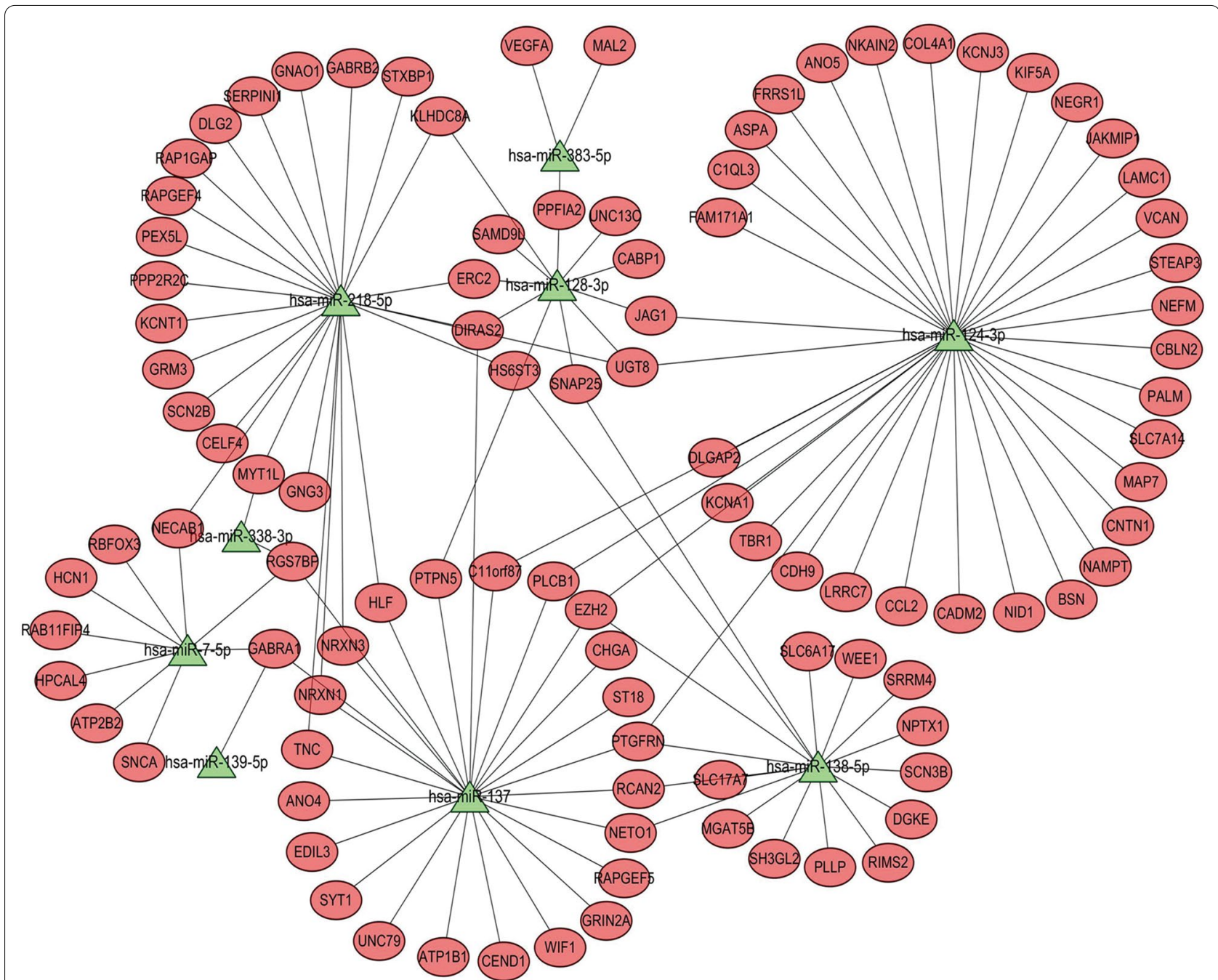

Fig. 4 The networks of validated DEMs and overlapping genes between DEGs and DEMs target genes. Red nodes represent upregulated factors, while green nodes represent downregulated factors. Triangles represent DEMs, and circles represent overlapping genes. DEMs differentially expressed miRNAs, DEGs differentially expressed genes

the miR-139-5p mimic on the invasive abilities of U251 and U87 cells (Figs. 9 and 10). In addition, cotransfection of the miR-139-5p inhibitor and OE-GABRA1 notably promoted migration and invasion compared with transfection of the miR-139-5p inhibitor alone. Cotransfection of the miR-139-5p inhibitor and $\mathrm{Si}$ GABRA1 significantly inhibited the migration and invasion of U251 and U87 cells (Figs. 9 and 10). Taken together, our data indicated that miR-139-5p modulates glioma cell migration and invasion by targeting GABRA1.

\section{MiR-139-5p regulates glioma cell proliferation} and apoptosis through targeting of GABRA 1

Cell proliferation and apoptosis experiments were then performed in U251 and U87 cell lines. The results of the quantitative measurement of EdU-positive cells indicated that downregulation of miR-139-5p promoted the proliferation, while upregulation of miR-139-5p inhibited the proliferation, of U251 and U87 cells (Fig. 11). Flow cytometric analysis showed that the cell apoptosis rate was notably higher in the miR-139-5p mimic group while the 


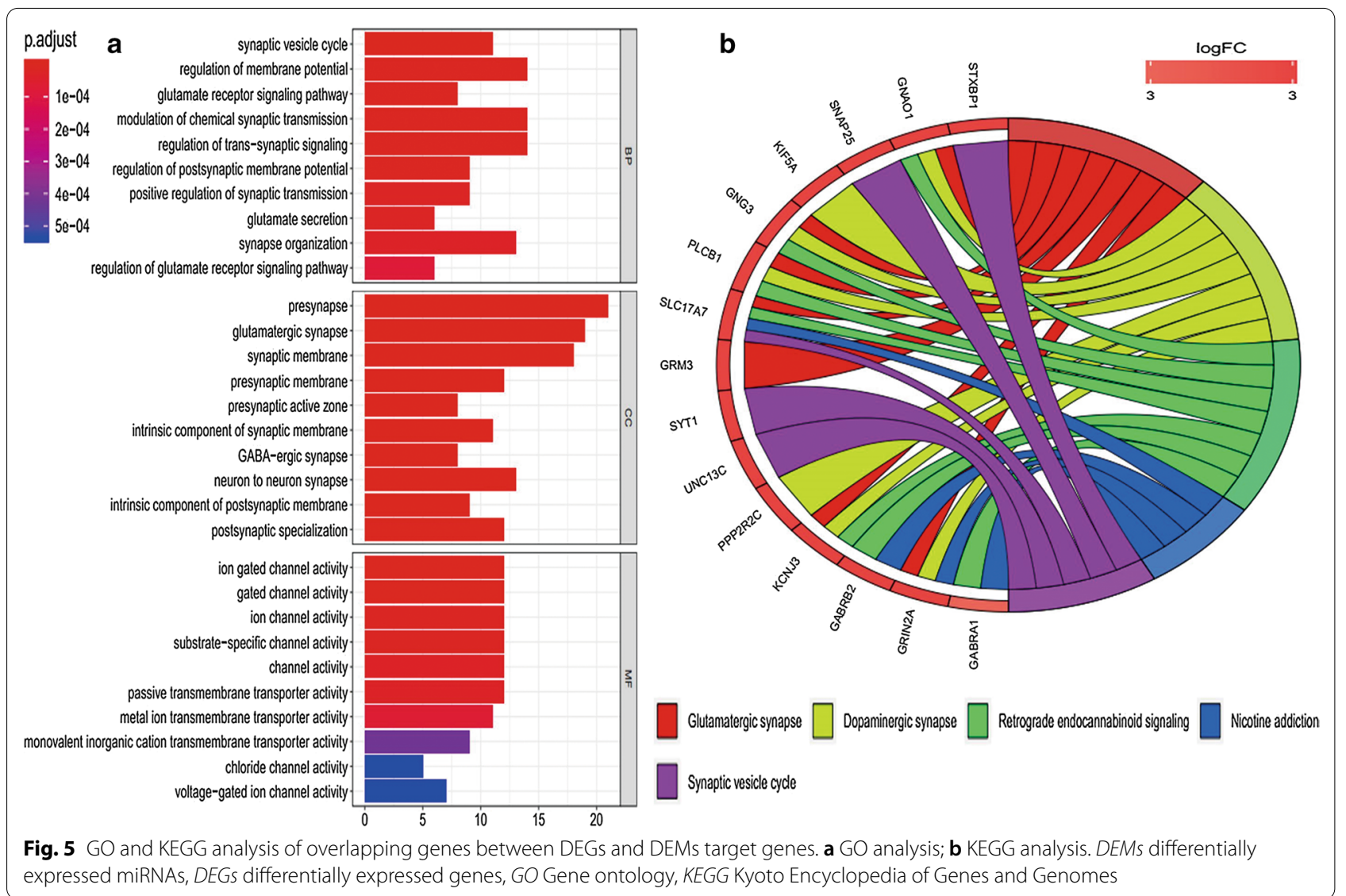

rate was lower in the miR-139-5p inhibitor group compared with the NC group, indicating that miR-139-5p induced the apoptosis of U251 and U87 cells (Fig. 12).

Since GABRA1 is directly targeted by miR-139-5p, we speculated that GABRA1 mediates the function of miR-139-5p. To test this hypothesis, we overexpressed GABRA1 in the miR-139-5p mimic group and found that GABRA1 overexpression rescued the miR-139-5pinduced inhibition of proliferation and increase in apoptosis (Figs. 11 and 12). Cotransfection of the miR-139-5p mimic and Si-GABRA1 significantly suppressed proliferation and promoted apoptosis, compared with transfection of miR-139-5p mimic alone (Figs. 11 and 12). The results revealed that the restoration of GABRA1 markedly reversed miR-139-5p-mediated glioma inhibitory effects.

\section{Discussion}

MiRNAs are a group of short and non-coding RNA molecules that influence the biological characteristics of GBM cells $[8,9]$. Mounting evidence has shown that miRNAs can act as cancer-suppressing factors as well as oncogenes [10-12]. MiRNAs play important roles in the tumorigenesis and progression, invasion, and metastasis of GBM by upregulating or downregulating cancer-related gene expression levels, and, therefore, have become promising biomarkers in the diagnosis and prognosis glioma [13].

In this work, bioinformatics technology was used to identify candidate miRNA biomarkers of glioblastoma via analysis of microarray data. As a result, 59 DEMs and 419 DEGs were identified in the GBM samples compared with healthy brain tissues. 
miR-137

\section{a All WHO Grade Survival (Primary Glioma)}

Strata $\rightarrow$ exp=High expression $(86)+$ exp=Low expression (86)

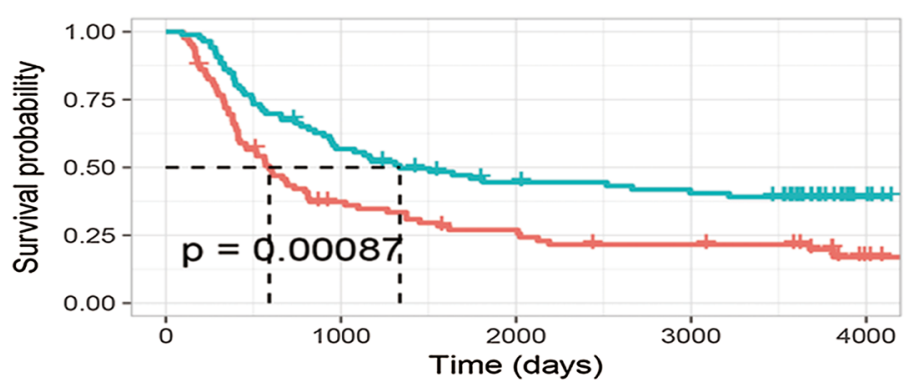

b

$\operatorname{miR}-338-3 p$ d

$20000-$

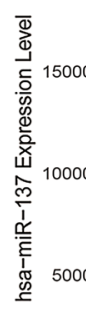

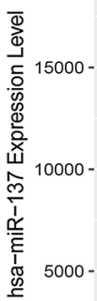

$0-$

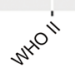

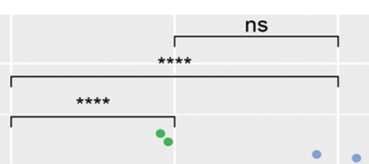
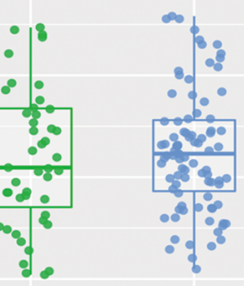

24:

WHO Grade

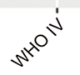

e

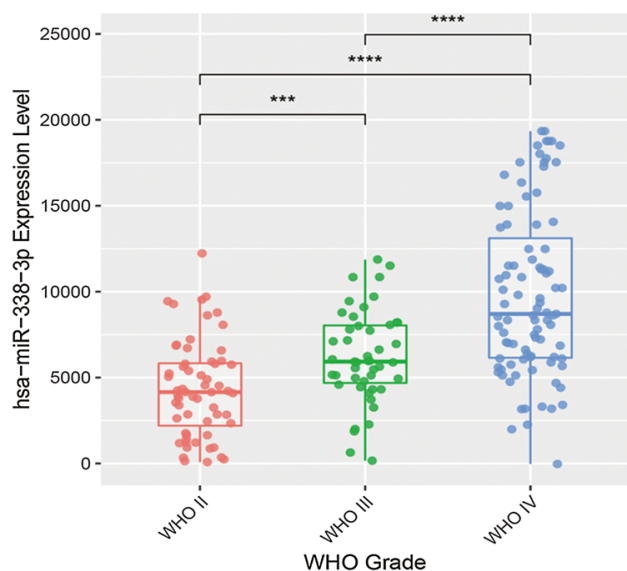

$\operatorname{miR}-139-5 p$

C

All WHO Grade Survival (Primary Glioma)

Strata + exp=High expression $(86)+\exp =$ Low expression (86)
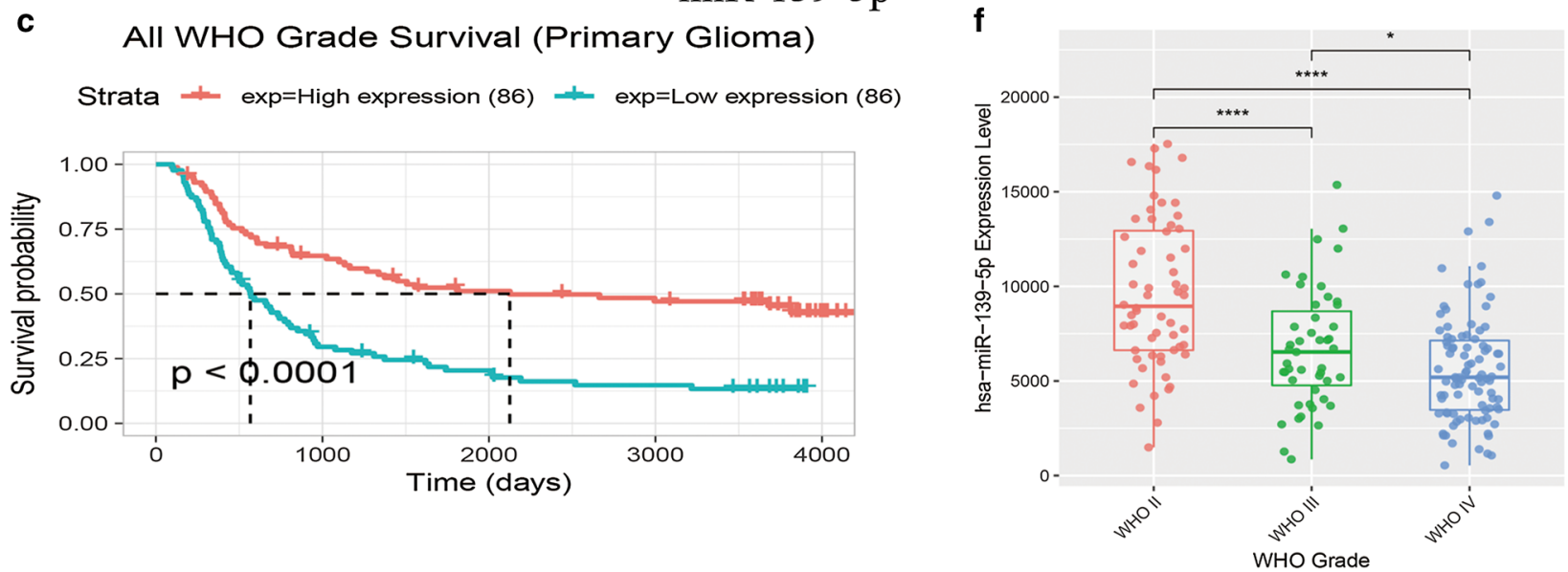

Fig. 6 The prognostic value of three DEMs in GBM and all grades glioma. The overall survival rate a Prognostic value of miR-137 in all grades glioma; b Prognostic value of miR-338-3p in all grades glioma; c Prognostic value of miR-139-5p in all grades glioma; d-e The expression of miR-137 and miR-338-3p was positively correlated with glioma grade. $\mathbf{f}$ the expression of miR-139-5p was negatively correlated with glioma grade 


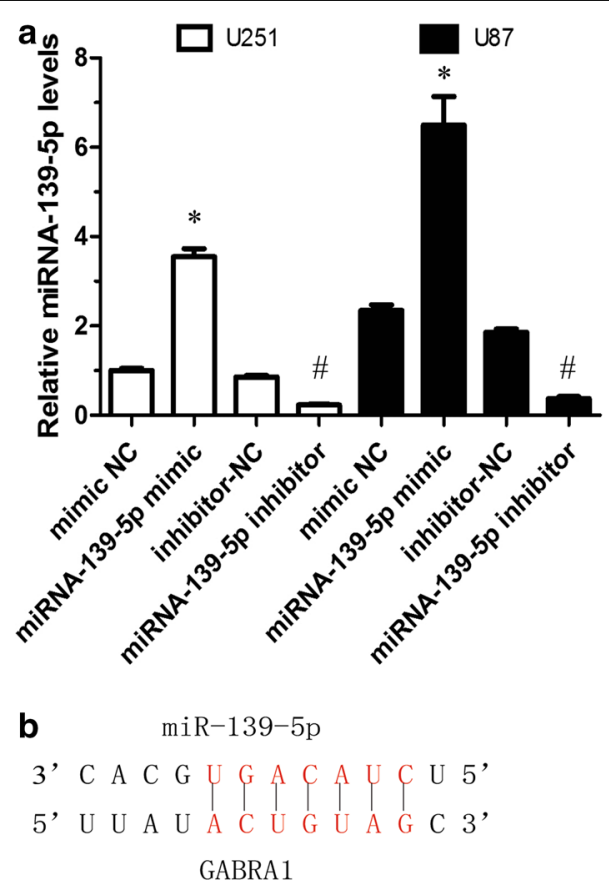

Fig. 7 a qRT-PCR analysis of GABRA1 mRNA expression in U251 and U87 cells transfected with miR-139-5p mimic or miR-139-5p inhibitor. b Putative miR-139-5p binding sites in the $3^{\prime}$-UTR sequence of GABRA1 genes. ${ }^{*} p<0.05$, vs mimic NC; \# $p<0.05$ vs inhibitor-NC

The prediction and GO enrichment analyses performed on the top ten DEMs showed that the majority of DEMs were enriched in signal transduction (BP) and transcription factor activity (MF), and were primarily located in the nucleus $(\mathrm{CC})$. To explore the interactions between DEMs and their corresponding target genes, we performed GO enrichment and KEGG pathway analyses using the list of targeted genes. GO term analysis indicated that the target genes were mainly involved in synaptic vesicle cycle and regulation of membrane potential. KEGG analysis of the target genes identified that they were involved in synaptic relevant pathways, including the glutamatergic synapse, dopaminergic synapse, and synaptic vesicle cycle signaling pathways. The results also indicated that the synaptic related genes aide in the prognosis of patients with glioma, which can improve objectivity in clinical judgment.

Subsequently, the integration analysis of the miRNAtarget gene regulatory pairs and DEGs revealed that 129 overlapping genes were regulated by nine DEMs. Further analysis revealed that miR-137, miR-139-5p, and miR338-3p were associated with prognosis in patients with all grades of glioma.

MiRNA-137 is an miRNA that is widely expressed in the central nervous system, and is particularly specific to hippocampal tissue [14]. It has been reported that the biological function of miRNAs may be related to synaptic plasticity and transmission $[15,16]$. Previous studies have indicated lower expression of miRNA-137 in tumor tissues, as compared with healthy tissues, in gastric cancer, colon cancer, and oral squamous cell carcinoma, suggesting that miRNA-137 acts as a suppressor $[17,18]$.

It has been reported that miR-338-3p functions as a tumor suppressor in several types of cancers [19-21]. Overexpression of miR-338-3p attenuated malignant biological behaviors of cells in gastric cancer and non-small cell lung cancer [19-21]. In the central nervous system, expression of miR-338-3p increases significantly as the dentate gyrus matures, and peaks in mature neurons [22]. Clinical data has also shown that low expression of miR$338-3 p$ corresponds to a decrease in overall survival and disease-free survival [22].

Here, we found differing conclusions from previous research based on data from the CGGA. Lower expression of miRNA-137 and miR-338-3p was found in glioma tissue than in peritumoral tissue, suggesting that these two miRNAs may be tumor suppressor genes, consistent with previously reported in the literature. However, in the CGGA data, high expression of miRNA-137 and miR-338-3p was also associated with poor overall survival in glioma patients. The expression of miRNA-137 and miR-338-3p were positively correlated with glioma grade, suggesting that both miRNAs might be cancerpromoting factors.

MiR-139-5p is considered as a cancer suppressor because its expression level is downregulated in several types of cancer, such as prostate [23], pancreatic [24], hepatocellular carcinoma [25, 26] and non-small cell lung cancer [27]. In GBM, miR-139-5p may suppress tumor cell invasion and migration via targeting ZEB1 and ZEB2 [28]. In addition, miR-139 has been shown to suppress glioma cell proliferation and enhance temozolomideinduced apoptosis [29]. 


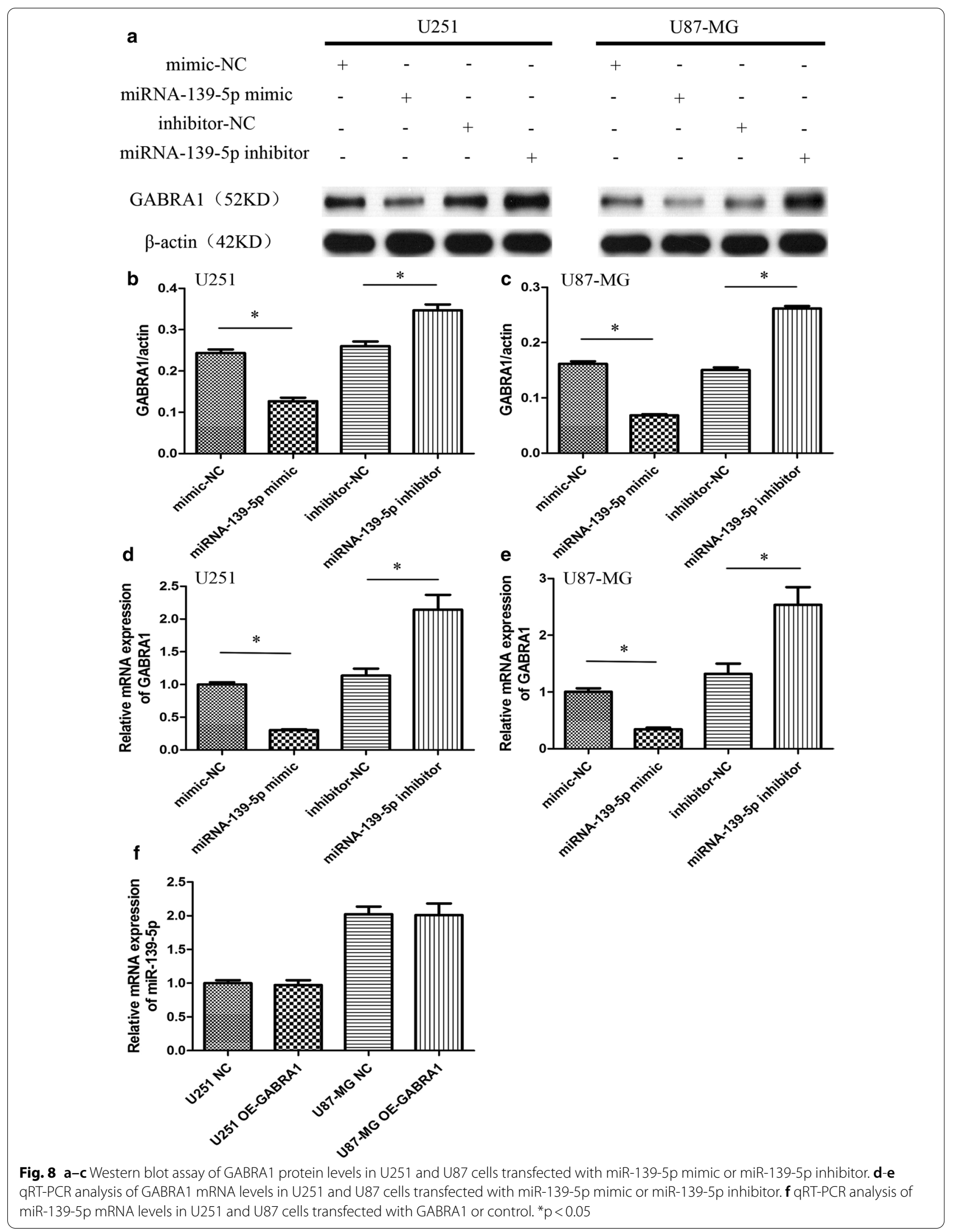



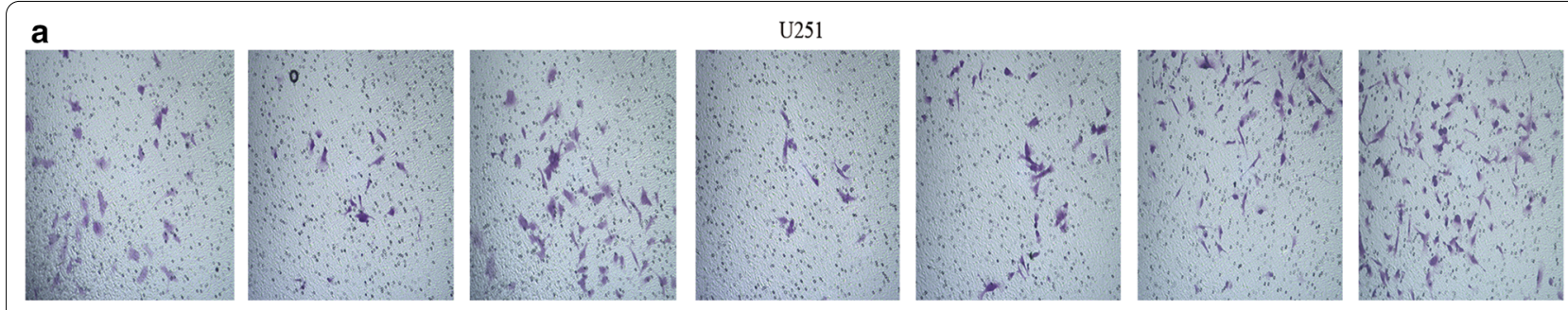

b
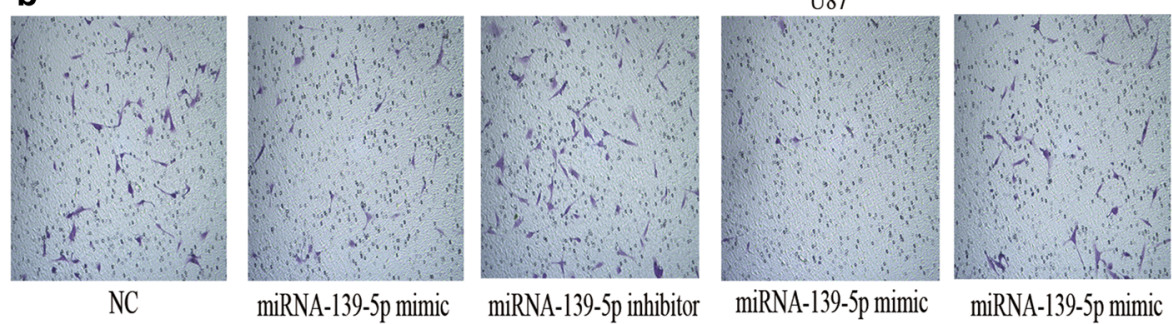

miRNA-139-5p mimic

miRNA-139-5p inhibitor

miRNA-139-5p mimic miRNA-139-5p mimic

Si-GABRAI OE-GABRAI
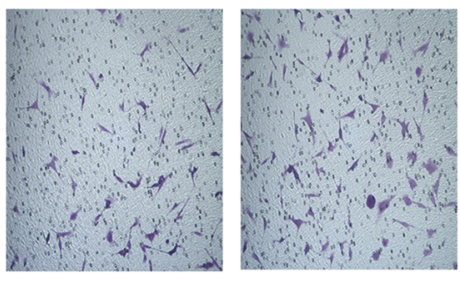
miRNA-139-5p inhibitor miRNA-139-5p inhibitor
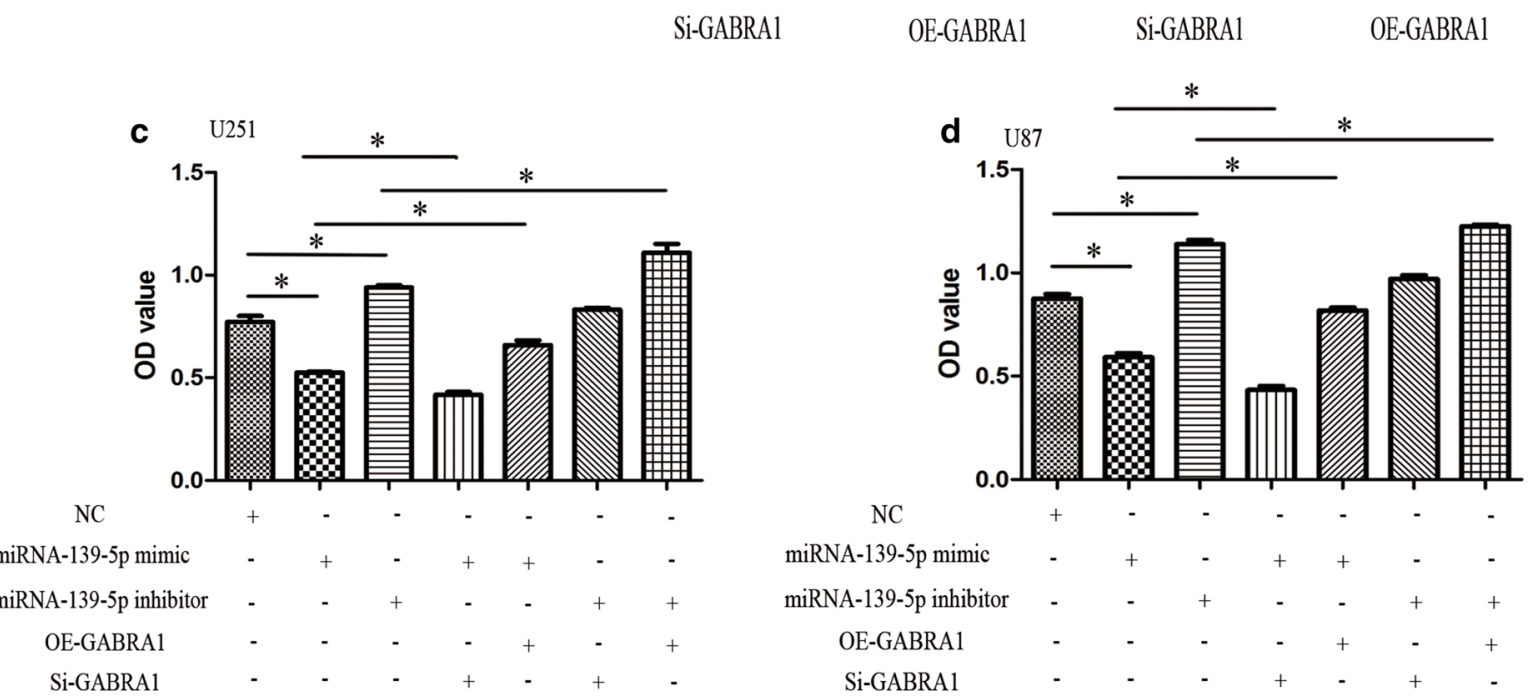

Fig. 9 Transwell assay in U251 and U87 cells after transfection with miRNA-139-5p mimic or miRNA-139-5p inhibitor and quantification of relative number of invaded cells. Each assay was repeated at least three times. ${ }^{*} \mathrm{P}<0.05$

More recently, several in depth studies have shown there are biophysical interactions between glioma cells and neurons, and that neurons can activate related neurotransmitter receptors through the synaptic connection with glioma cells to promote the growth of glioma cells. [30, 31]. Gamma-aminobutyric acid(GABA), the most common inhibitory neurotransmitter in the brain, binds to the GABA-A channel. The GABA-A channel consists of subunit isoforms (two -subunits, two -subunits, and a third type of subunit), and is the most common type of GABA receptor. [32, 33]. The GABRA1 subunit is highly expressed in the central nervous system, as is one of the key subunits of GABA receptors. There are few studies on the relationship between GABRA1 expression and gliomas, and any that do exist have been controversial in regard to the expression level of GABRA1 in gliomas. D'Urso's study indicated a lack of GABRA1 mRNA expression in glioblastoma primary cultures via northern blot and immunohistochemical analysis [34]. Another study showed that GABRA1 was expressed in gliomas, and that the expression level was highest in WHO grade II gliomas [35]. The effect of GABRA1 expression on the 

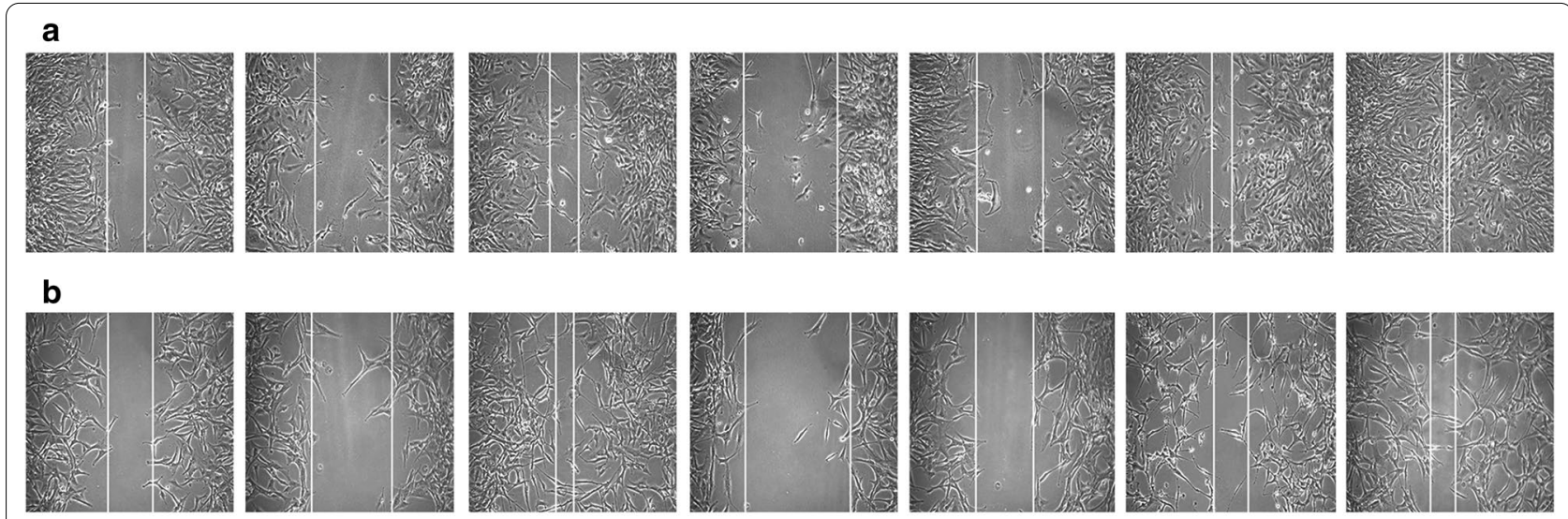

NC

miRNA-139-5p mimic miRNA-139-5p inhibitor

miRNA-139-5p mimic

miRNA-139-5p mimic

miRNA-139-5p inhibitor miRNA-139-5p inhibitor

Si-GABRAl OE-GABRAl

Si-GABRAl

OE-GABRAl

C

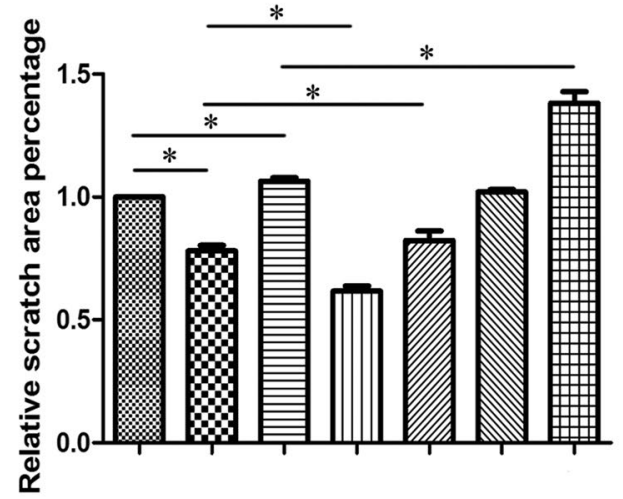

$\mathrm{NC}$ d

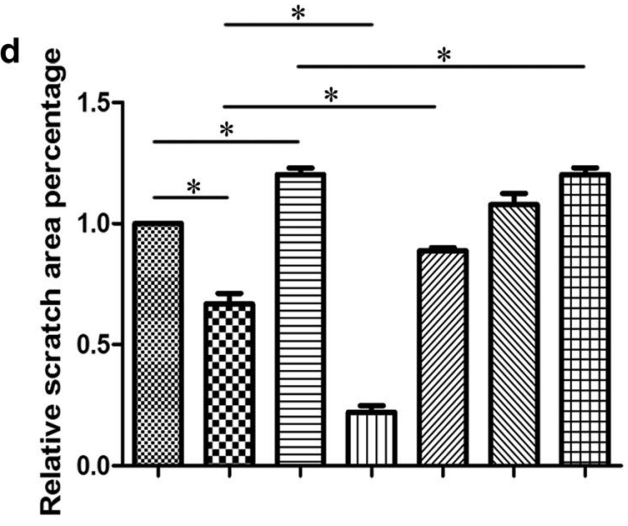

$\mathrm{NC}$

$$
\begin{array}{cccccccr}
\text { miRNA-139-5p mimic } & - & + & - & + & + & - & - \\
\text { miRNA-139-5p inhibitor } & - & - & + & - & - & + & + \\
\text { OE-GABRA1 } & - & - & - & - & + & - & + \\
\text { Si-GABRA1 } & - & - & - & + & - & + & -
\end{array}
$$

Fig. 10 Wounded healing assay in U251 and U87 cells after transfection with miRNA-139-5p mimic or miRNA-139-5p inhibitor and quantification of relative scratch area. Each assay was repeated at least three times. ${ }^{*} \mathrm{P}<0.05$

malignant biological behavior of glioma has not been reported. Available literature suggests that endogenous GABA receptor activity within glioma cells has a significant impact on tumor development [36]. Our experimental results in vitro suggest that GABRA1 is expressed in the glioma cell lines U251 and U87, and through overexpression and silencing experiments, we found that GABRA1 plays a role in promoting the growth of gliomas, which is directly regulated by miR-139-5p. 


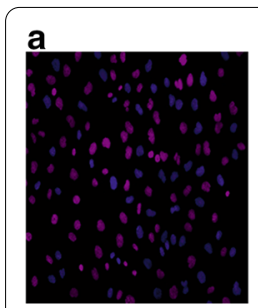

b

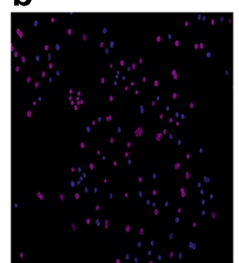

$\mathrm{NC}$
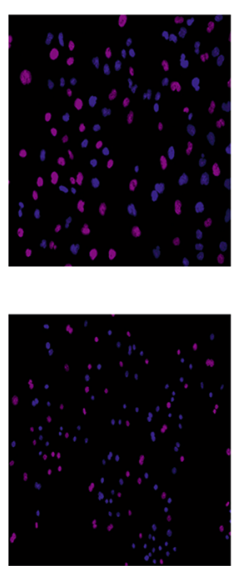

miRNA-139-5p mimic
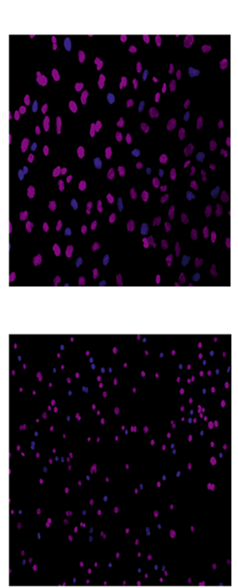

miRNA-139-5p inhibitor

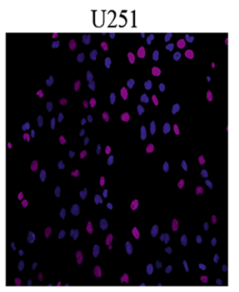

U87

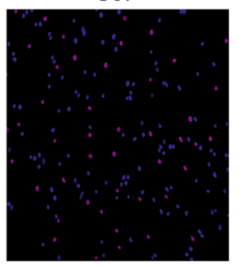

miRNA-139-5p mimic Si-GABRAl
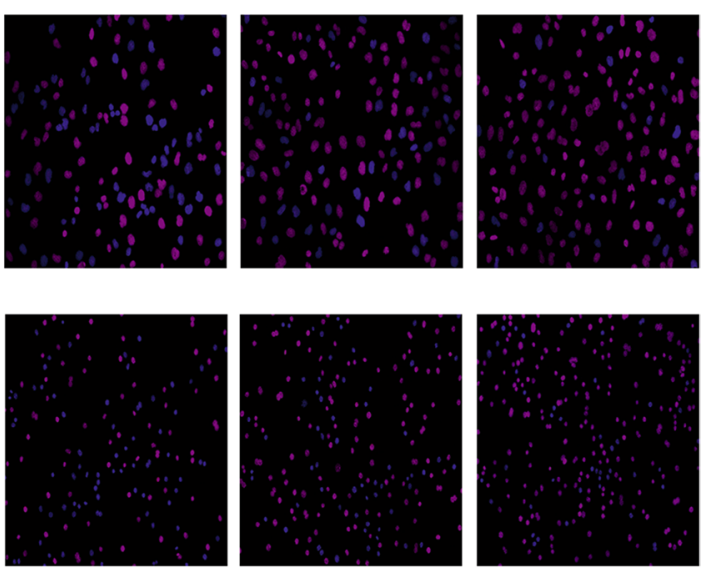

miRNA-139-5p mimic

OE-GABRAl

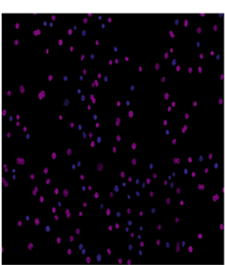

miRNA-139-5p inhibitor miRNA-139-5p inhibitor Si-GABRA1

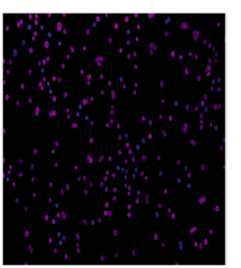

OE-GABRAl

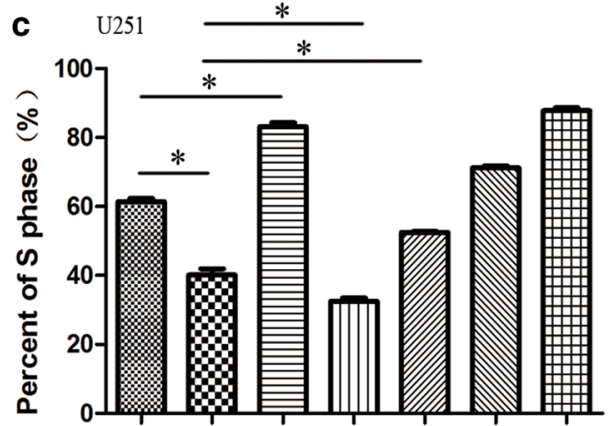

$\mathrm{NC}$

miRNA-139-5p mimic

miRNA-139-5p inhibitor

OE-GABRA1

Si-GABRA1

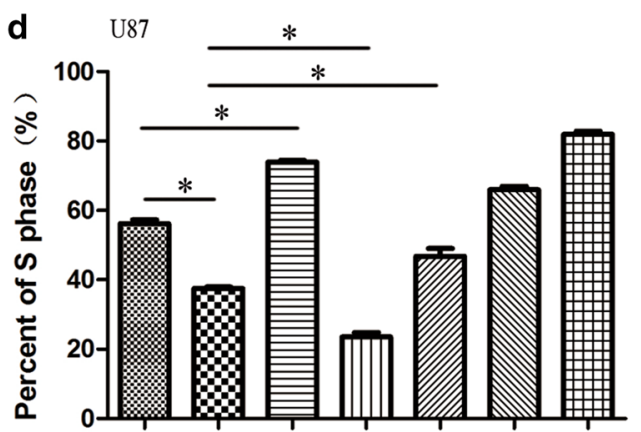

$\mathrm{NC}$

miRNA-139-5p mimic

miRNA-139-5p inhibitor

OE-GABRA1

Si-GABRA1

Fig. 11 EdU assay in U251 and U87 cells after transfection with miRNA-139-5p mimic or miRNA-139-5p inhibitor and quantification of S phase. Each assay was repeated at least three times. ${ }^{*} \mathrm{P}<0.05$

\section{Conclusions}

In conclusion, we analyzed a combination of core miRNAs and target gene expressions between GBM and peritumoral tissue to further identify a set of promising diagnostic and prognostic biomarkers. We showed that miR-139-5p expression levels were decreased in glioma samples and that the miR-139-5p expression was significantly negatively correlated with prognosis of glioma patients. In addition, we discovered that miR-139-5p expression had a negative correlation with GABRA1 expression in U251 and U87 cells. Moreover, we found that miR-139-5p exerted tumor anti-tumor functions in glioma by directly targeting GABRA1. 

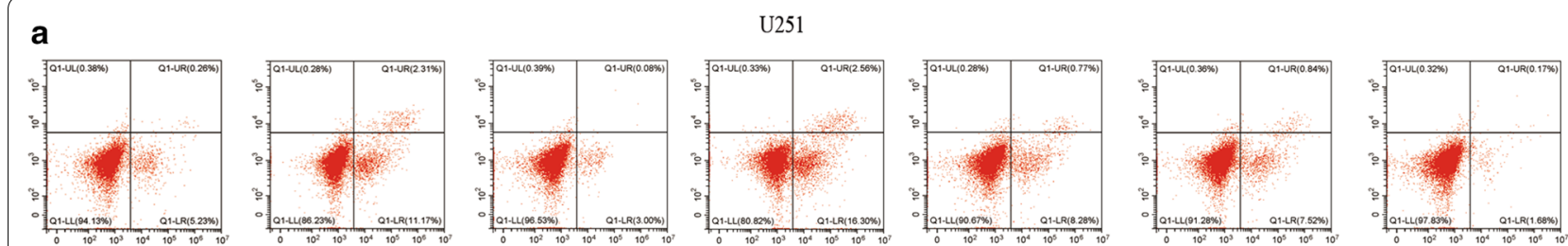

b
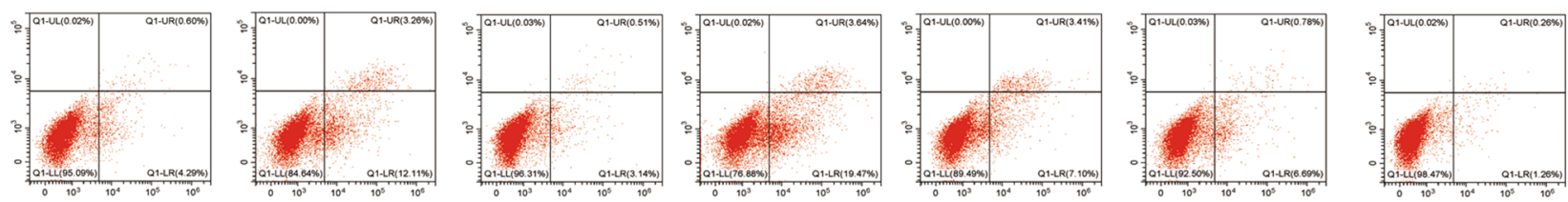

$\mathrm{NC}$

miRNA-139-5p mimic miRNA-139-5p inhibitor

miRNA-139-5p mimic

miRNA-139-5p mimic

miRNA-139-5p inhibitor

miRNA-139-5p inhibitor

Si-GABRAl

OE-GABRAl

Si-GABRAl

OE-GABRAl
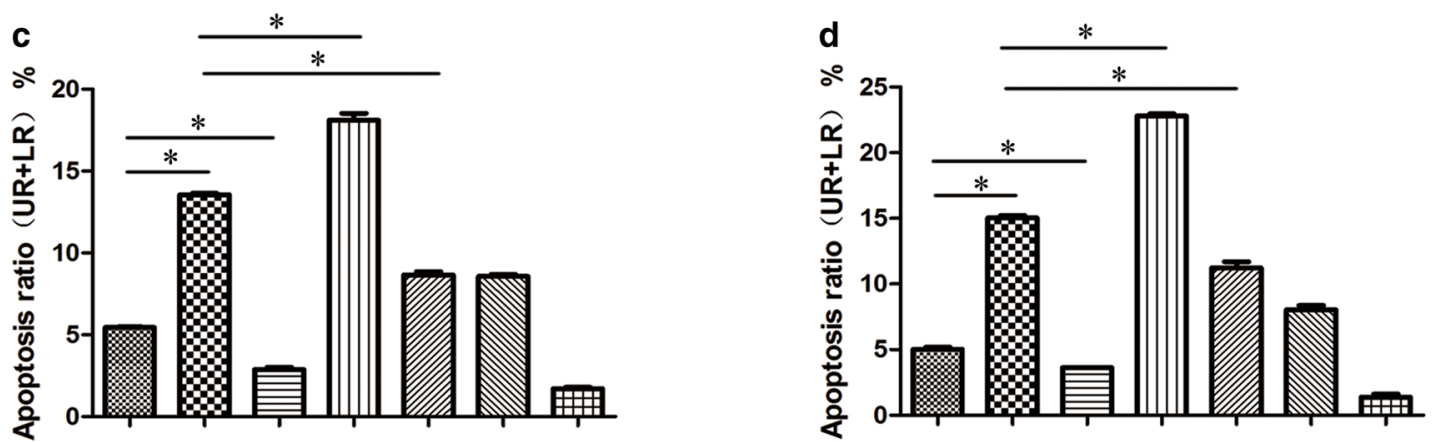

$\mathrm{NC}$

miRNA-139-5p mimic

miRNA-139-5p inhibitor

OE-GABRA1

Si-GABRA1

Fig. 12 Flow cytometry assay in U251 and U87 cells after transfection with miRNA-139-5p mimic or miRNA-139-5p inhibitor and quantification of apoptosis ratio. Each assay was repeated at least three times. ${ }^{*} \mathrm{P}<0.05$

\section{Supplementary Information}

The online version contains supplementary material available at https://doi. org/10.1186/s12967-021-02880-9.

Additional file 1: All DEMs between GBM sample compared with normal brain tissues.

Additional file 2: All DEGs between GBM sample compared with normal brain tissues.

Additional file 3: $G O$ enrichment of DEMs(BP)

Additional file 4: GO enrichment of DEMs(CC)

Additional file 5: $G O$ enrichment of DEMs(MF)

Additional file 6: $\mathrm{GO}$ analysis of the overlapping target genes

Additional file 7: KEGG analysis of the overlapping target genes

\section{Acknowledgements}

We gratefully acknowledge technical support from Well Biological Science company. We gratefully acknowledge professional writing services support form International Science Editing.

\section{Authors' contributions}

$L W$ and $Z H$ designed experiments and directed the project. $Z Y$ and $Y L$ performed experiments.JG and ZD analysed experiments and drafted the manuscript. NR, ZZ, HC, ZT, HC and SC performed data analysis. All the authors reviewed the manuscript. All authors read and approved the final manuscript.

\section{Funding}

This study was supported by the Hunan Provincial Natural Science Foundation of China (No.2019JJ40182), Scientific Research Project of Hunan Provincial Health Commission (No. 20200709), Hunan Cancer Hospital Climb Plan (No. QH201906 and 2020NSFC-B009) and Hainan Provincial Natural Science Foundation of China (No.820MS163).

\section{Availability of data and materials}

The datasets used or analyzed during the current study are available from The Chinese Glioma Genome Atlas (http://www.cgga.org.cn/).

\section{Declarations}

Ethics approval and consent to participate

Not applicable. 


\section{Consent for publicationConsent for publication}

Not applicable.

\section{Competing interests}

The authors declare that they have no competing interests.

\begin{abstract}
Author details
${ }^{1}$ Department of Neurosurgery, Hunan Cancer Hospital and The Affiliated Cancer Hospital of Xiangya School of Medicine, Central South University, No.283 Tongzipo road, Yuelu district, Changsha 410006, Hunan, China. ${ }^{2}$ Department of Neurology, Changsha Central Hospital, University of South China, No.161 Shaoshan road, Yuhua district, Changsha 410007, Hunan, China. ${ }^{3}$ Department of Neurosurgery, Haikou People's Hospital, The Affiliated Haikou Hospital of Xiangya School of Central South University, No.43 Renmin road, Meilan district, Haikou 570208, Hainan, China.
\end{abstract}

Received: 20 February 2021 Accepted: 8 May 2021

Published online: 17 May 2021

\section{References}

1. Louis DN, Ohgaki H, Wiestler OD, Cavenee WK, Burger PC, Jouvet A, et al. The 2007 WHO classification of tumours of the central nervous system. Acta Neuropathol. 2007;114:97-109.

2. Sadetzki S, Zach L, Chetrit A, Nass D, Hoffmann C, Ram Z, et al. Epidemiology of gliomas in Israel: a nationwide study. Neuroepidemiology. 2008:31:264-9.

3. Huang J, Samson P, Perkins SM, Ansstas G, Chheda MG, DeWees TA, et al. Impact of concurrent chemotherapy with radiation therapy for elderly patients with newly diagnosed glioblastoma: a review of the National Cancer Data Base. J Neurooncol. 2017;131:593-601.

4. Anton K, Baehring JM, Mayer T. Glioblastoma multiforme: overview of current treatment and future perspectives. Hematol Oncol Clin North Am. 2012:26:825-53.

5. Qiu S, Lin S, Hu D, Feng Y, Tan Y, Peng Y. Interactions of miR-323/miR326/miR-329 and miR-130a/miR-155/miR-210 as prognostic indicators for clinical outcome of glioblastoma patients. J Transl Med. 2013;11:10.

6. Zhou Q, Liu J, Quan J, Liu W, Tan H, Li W. MicroRNAs as potential biomarkers for the diagnosis of glioma: A systematic review and metaanalysis. Cancer Sci. 2018;109:2651-9.

7. Pathan M, Keerthikumar S, Ang CS, Gangoda L, Quek CY, Williamson NA, et al. FunRich: An open access standalone functional enrichment and interaction network analysis tool. Proteomics. 2015;15:2597-601.

8. Zhou F, Cao W, Xu R, Zhang J, Yu T, Xu X, et al. MicroRNA-206 attenuates glioma cell proliferation, migration, and invasion by blocking the WNT/ $\beta$-catenin pathway via direct targeting of Frizzled 7 mRNA. Am J Transl Res. 2019;11:4584-601.

9. Xin, S., Huang, K., Zhu, X. G., Non-coding RNAs: Regulators of glioma cell epithelial-mesenchymal transformation, Pathol. Res. Pract. (2019) 215:152539.

10. Yang H, Song Z, Wu X, Wu Y, Liu C. MicroRNA-652 suppresses malignant phenotypes in glioblastoma multiforme via FOXK1-mediated AKT/ mTOR signaling pathway. Onco Targets Ther. 2019;12:5563-75.

11. Zuo J, Yu H, Xie P, Liu W, Wang K, Ni H. miR-454-3p exerts tumor-suppressive functions by down-regulation of NFATc2 in glioblastoma. Gene. 2019:710:233-9.

12. Wu W, Yu T, Wu Y, Tian W, Zhang J, Wang Y. The miR155HG/miR-185/ ANXA2 loop contributes to glioblastoma growth and progression. J Exp Clin Cancer Res. 2019;38:133.

13. Nikaki A, Piperi C, Papavassiliou AG. Role of microRNAs in gliomagenesis: targeting miRNAs in glioblastoma multiforme therapy. Expert Opin Investig Drugs. 2012;21:1475-88.

14. Yin J, Lin J, Luo X, Chen Y, Li Z, Ma G, et al. miR-137: a new player in schizophrenia. Int J Mol Sci. 2014;15:3262-71.

15. Kos A, Aschrafi A, Nadif KN. The multifarious hippocampal functions of MicroRNA-137. Neuroscientist. 2016;22:440-6.

16. Wu L, Chen J, Ding C, Wei S, Zhu Y, Yang W, et al. MicroRNA-137 Contributes to Dampened Tumorigenesis in Human Gastric Cancer by Targeting AKT2. PLOS ONE. 2015:10:e0130124.
17. Sakaguchi M, Hisamori S, Oshima N, Sato F, Shimono Y, Sakai Y. miR-137 regulates the tumorigenicity of colon cancer stem cells through the inhibition of DCLK1. Mol Cancer Res. 2016;14:354-62.

18. Sun C, Li J. Expression of MiRNA-137 in oral squamous cell carcinoma and its clinical significance. J BUON. 2018;23:167-72.

19. Zhang R, Shi H, Ren F, Liu Z, Ji P, Zhang W, et al. Down-regulation of miR-338-3p and Up-regulation of MACC1 indicated poor prognosis of epithelial ovarian cancer patients. J Cancer. 2019;10:1385-92.

20. Zhang P, Shao G, Lin X, Liu Y, Yang Z. MiR-338-3p inhibits the growth and invasion of non-small cell lung cancer cells by targeting IRS2. Am J Cancer Res. 2017;7:53-63.

21. Sun F, Yu M, Yu J, Liu Z, Zhou X, Liu Y, et al. miR-338-3p functions as a tumor suppressor in gastric cancer by targeting PTP1B. Cell Death Dis. 2018:9:522.

22. Howe JR, Li ES, Streeter SE, Rahme GJ, Chipumuro E, Russo GB, et al. MiR-338-3p regulates neuronal maturation and suppresses glioblastoma proliferation. PLoS ONE. 2017;12:e0177661.

23. Nam RK, Benatar T. CJD, W, Kobylecky, E, Amemiya, Y, Sherman, C, et al, MicroRNA-139 is a predictor of prostate cancer recurrence and inhibits growth and migration of prostate cancer cells through cell cycle arrest and targeting IGF1R and AXL. Prostate. 2019;79:1422-38.

24. Bai $X, L u ~ D, L i n Y, L v Y, H e ~ L . ~ A ~ s e v e n-m i R N A$ expression-based prognostic signature and its corresponding potential competing endogenous RNA network in early pancreatic cancer. Exp Ther Med. 2019;18:1601-8.

25. Wang X, Gao J, Zhou B, Xie J, Zhou G, Chen Y. Identification of prognostic markers for hepatocellular carcinoma based on miRNA expression profiles. Life Sci. 2019;232:116596.

26. Li P, Xiao Z, Luo J, Zhang Y, Lin L. MiR-139-5p, miR-940 and miR-193a-5p inhibit the growth of hepatocellular carcinoma by targeting SPOCK1. J Cell Mol Med. 2019;23:2475-88.

27. Yong-Hao Y, Xian-Guo W, Ming X, Jin-Ping Z. Expression and clinical significance of miR-139-5p in non-small cell lung cancer. J Int Med Res. 2019:47:867-74.

28. Yue S, Wang L, Zhang H, Min Y, Lou Y, Sun H, et al. miR-139-5p suppresses cancer cell migration and invasion through targeting ZEB1 and ZEB2 in GBM. Tumour Biol. 2015;36:6741-9.

29. Li RY, Chen LC, Zhang HY, Du WZ, Feng Y, Wang HB, et al. MiR-139 inhibits $\mathrm{MCl}-1$ expression and potentiates TMZ-induced apoptosis in glioma. CNS Neurosci Ther. 2013;19:477-83

30. Venkataramani V, Tanev DI, Strahle C, Studier-Fischer A, Fankhauser L, Kessler T, et al. Glutamatergic synaptic input to glioma cells drives brain tumour progression. Nature. 2019:573:532-8.

31. Venkatesh HS, Morishita W, Geraghty AC, Silverbush D, Gillespie SM, Arzt $M$, et al. Electrical and synaptic integration of glioma into neural circuits. Nature. 2019;573:539-45.

32. Birnir B, Korpi ER. The impact of sub-cellular location and intracellular neuronal proteins on properties of GABA(A) receptors. Curr Pharm Des. 2007;13:3169-77.

33. Young SZ, Bordey A. GABA's control of stem and cancer cell proliferation in adult neural and peripheral niches. Physiology (Bethesda). 2009:24:171-85.

34. D'Urso PI, D'Urso OF, Storelli C, Mallardo M, Gianfreda CD, Montinaro A, et al. miR-155 is up-regulated in primary and secondary glioblastoma and promotes tumour growth by inhibiting GABA receptors. Int J Oncol. 2012:41:228-34.

35. Smits A, Jin Z, Elsir T, Pedder H, Nistér M, Alafuzoff I, et al. GABA-A channel subunit expression in human glioma correlates with tumor histology and clinical outcome. PLoS ONE. 2012;7:e37041.

36. Blanchart A, Fernando R, Häring M, Assaife-Lopes N, Romanov RA, Andäng $M$, et al. Endogenous GABAA receptor activity suppresses glioma growth. Oncogene. 2017;36:777-86.

\section{Publisher's Note}

Springer Nature remains neutral with regard to jurisdictional claims in published maps and institutional affiliations. 